\title{
CONTRIBUIÇÃO DE DIVERSOS SISTEMAS DE OBSERVAÇÃO NA PREVISÃO DE TEMPO NO CPTEC/INPE
}

\author{
RITA V. ANDREOLI, SÉRGIO HENRIQUE S. FERREIRA, LUIZ F. SAPUCCI, \\ RODRIGO AUGUSTO F. DE SOUZA,RENATA WEISSMANN B. MENDONÇA, \\ DIRCEU L. HERDIES, JOSÉ A. ARAVÉQUIA \\ Instituto Nacional de Pesquisas Espaciais (INPE) \\ Centro de Previsão de Tempo e Estudos Climáticos (CPTEC) \\ rita@cptec.inpe.br
}

Recebido Junho 2007 - Aceito Fevereiro 2008

\begin{abstract}
RESUMO
Experimentos utilizando sistemas de observação global, foram realizados excluindo um ou mais tipos de observação do esquema global de assimilação de dados/previsão de tempo do Centro de Previsão de Tempo e Estudos Climáticos do Instituto Nacional de Pesquisas Espaciais - CPTEC/INPE (Global Physical-space Statistical Analysis System - GPSAS). Estes experimentos indicam como efetivamente as observações são usadas no GPSAS. Os sistemas de observação testados foram o conjunto de dados convencionais, que incluem informações de superfície (estações em superfície, bóias, navios e plataformas oceânicas) e de ar superior (radiossondagem, aeronaves e balões piloto), os sistemas de sondagem Advanced TIROS-N/NOAA Operational Vertical Sounder (ATOVS) e AQUA, composto pelos sensores Atmospheric Infrared Sounder e Advanced Microwave Sounding Unit (AIRS/AMSU), dados de vento de satélite, estimados a partir do deslocamento de nuvens (Cloud Track Wind), dados de vento em superfície sobre o oceano (QuikScat) e água precipitável (Total Precipitation water - TPW). Todos os sistemas testados mostram um impacto positivo na qualidade da previsão. Os dados convencionais têm um maior impacto na região do Hemisfério Norte devido à maior disponibilidade dessas informações sobre esta região. Por outro lado, as sondagens AIRS/AMSU são fundamentais para uma boa previsão sobre o Hemisfério Sul. Sobre a América do Sul, os perfis inferidos pelo sistema de sondagem AQUA contribuem com a mesma ordem de grandeza dos dados convencionais e apresentam um impacto positivo para todos os períodos de previsões analisados. Dados de vento e água precipitável estimados por satélites têm maior impacto nas regiões tropical e da América do Sul, nas primeiras horas de previsão (1-3 dias). Todavia, a utilização de um conjunto completo de observações é crucial para se obter, operacionalmente, uma boa condição inicial do estado atmosférico para ser utilizada nos modelos de previsão numérica de tempo do CPTEC/INPE. Palavras Chave: Sistema de observação, Assimilação de dados, PSAS, Previsão Numérica de Tempo.
\end{abstract}

\footnotetext{
ABSTRACT: CONTRIBUTION OF THE SEVERAL OBSERVATION SYSTEMS IN THE FORECAST SKILL AT CPTEC/INPE.

A series of data withholding experiments was conducted at Center for Weather Forecasting and Climate Studies of the National Institute for Space Research (CPTEC/INPE), with the Global Physicalspace Statistical Analysis System (GPSAS) - a combination of the Spectral Atmospheric Global Circulation Model (CPTEC/COLA) with the Physical-space Statistical Analysis System (PSAS). These experiments indicate how efficiently the observations are used in the particular assimilation system. The observation tested were from conventional data (SYNOP, BUOY, SHIP, radiosonde, aircraft, pilot balloons), and satellite data (ATOVS and AIRS/AMSU retrievals, QuikScat wind, Cloud Track Wind and Total Precipitation Water from SSM-I sensor). All observing systems tested have a notable positive
} 
impact on the forecast performance. The conventional data have a large impact on North Hemisphere region and it is associated the high density of these information in this region. On the other hand, the AIRS/AMSU retrievals had the largest impact in the Southern Hemisphere. Over the South America, AIRS/AMSU retrievals contribute with the same order of magnitude as conventional data and have a positive impact on all range forecast (1-5 day). Wind and TPW satellite-derived data had the largest impact in the tropical and South America regions at short (1-3 day) range forecast. However, the use of the major observing system it is crucial to obtain operationally a good initial condition of the atmospheric state for initializing of the numerical weather models at CPTEC/INPE.

Keywords: observation system, data assimilation, PSAS, weather prediction

\section{INTRODUÇÃO}

O Centro de Previsão de Tempo e Estudos Climáticos do Instituto Nacional de Pesquisas Espaciais (CPTEC/INPE) executa modelos numéricos de previsões de tempo em escala global e regional com a finalidade de produzir, operacionalmente, previsões de tempo para o Brasil. Atualmente, essas previsões, produzidas especialmente a partir de modelos globais, são realizadas utilizando condições iniciais (análises) geradas no National Center For Enviroment Pediction (NCEP). Paralelamente, esforços têm sido realizados com o intuito de gerar, localmente, as condições iniciais de forma operacional. Para isso, faz-se necessário a utilização de um sistema de assimilação de dados, no qual as observações atmosféricas em um determinado instante e em localidades diversas, obtidas a partir de várias fontes de informação (convencionais ou derivadas por satélites), são coletadas e avaliadas para produzir uma análise (condição inicial) que representa, no espaço do modelo numérico, o estado atmosférico naquele instante. No CPTEC, o método de assimilação de dados utilizado é o Physical-space Statistical Analysis System (PSAS, Cohn et al., 1998). O PSAS, usado operacionalmente no CPTEC, é algebricamente equivalente a outros esquemas 3D-Var, tais como o Spectral Statistical Interpolation (SSI) do NCEP (Parrish e Derber, 1992) e o 3D-Var do European Centre for MediumRange Weather Forecasts - ECMWF (Coutier et al., 1998), mas difere destes esquemas no fato que este é formulado diretamente no espaço das observações ao invés do espaço do modelo (Cohn et al., 1998). Todavia, sabe-se que um dos fatores que determinam o desempenho dos sistemas de assimilação de dados está relacionado à quantidade e qualidade das observações. Neste contexto, esforços têm sido direcionados no sentido de incorporar mais observações no sistema de assimilação de dados/previsão de tempo do CPTEC, a fim de se obter operacionalmente, uma condição inicial mais realística possível para os modelos de PNT.

Nos últimos anos, vários avanços foram realizados no que diz respeito à inclusão de novas observações no sistema de assimilação de dados no CPTEC, principalmente, àquelas relacionadas às informações estimadas por satélites (Cintra et al., 2005, Andreoli et al., 2006, Sapucci et al. 2006, Ferreira e Aravéquia, 2006). Dentre esses estudos, Andreoli et al. 2006 mostraram que a inclusão dos perfis atmosféricos inferidos pelo sistema de sondagem do satélite AQUA, composto pelos sensores Atmospheric Infrared Sounder e Advanced Microwave Sounding Unit (AIRS/AMSU), no sistema de assimilação/ previsão aponta para uma melhora tanto nos campos de análises quanto nos de previsões, principalmente, em regiões onde o número de observações convencionais é baixo. A melhora no desempenho da previsão de 4 dias para o Hemisfério Sul foi equivalente a ganhar uma extensão da capacidade de previsão em torno de 6 horas. Este resultado indicou o considerável potencial das sondagens AIRS/AMSU para melhorar a qualidade das análises e previsões de tempo de médio prazo geradas no CPTEC. Por outro lado, Ferreira e Aravéquia (2006) mostraram que o impacto das informações de vento estimadas por satélite, a partir do deslocamento de nuvens, ocorre nas primeiras horas de previsão.

Os estudos citados anteriormente buscaram avaliar o impacto de um sistema de observação isoladamente, e utilizaram diferentes conjuntos de análises para verificação. Este trabalho tem por objetivo avaliar o impacto dos diversos sistemas de observação no esquema de assimilação/previsão de tempo global do CPTEC utilizando um campo de análise comum para todos os experimentos. Geralmente, estudos de impacto são realizados através de experimentos nos quais, um sistema de observação é retido no processo de assimilação (Zapotocny et al., 2005) ou com a avaliação do impacto através de experimentos nos quais adiciona-se observações à um esquema de assimilação/ previsão pré-definido. Outra maneira de se avaliar o impacto de vários sistemas de observação no desempenho dos modelos numéricos é através de estudos de casos individuais de padrões sinóticos (Cavalcanti e Herdies, 2004; Chen et al., 2004; Atlas et al., 2001). Estudos dessa natureza fornecem um completo entendimento de como utilizar as informações dos diversos sistemas de observações já existentes de forma eficiente e são essenciais para o aprimoramento da qualidade das análises e das previsões numéricas de tempo. Consequentemente, estudos que 
avaliam o impacto dos diversos sistemas de observação têm sido realizados em vários centros de previsão numérica de tempo. Dentre esses estudos, citam-se: Paullex et al. 1997; Bouttier e Kelly, 2001; Verner at al. 2004, Kelly et al., 2004; Zapotocny et al., 2005, entre outros.

Em geral, esses estudos mostram a importância de se utilizar dados de satélites, principalmente em regiões onde existe uma escassez de dados convencionais. Além disso, observações de satélite que foram durante algum tempo o principal contribuinte de informações sobre o Hemisfério Sul, são consideradas mais recentemente, como fonte primária de informações para modelos de PNT para todo o globo. Este fato está relacionado à melhoria dos produtos de satélite, associada aos avanços nos instrumentos e nos sistemas de préprocessamento, bem como ao crescente desenvolvimento e aperfeiçoamento dos sistemas de assimilação de dados.

Com o intuito de avaliar o impacto de sistemas de observação na previsão numérica de tempo gerada no CPTEC, neste estudo é realizada uma série de experimentos utilizando diversos sistemas de observações. Esta série de experimentos é na realidade o primeiro conjunto de experimentos realizado no CPTEC, no qual se utilizam diferentes sistemas de observação que estão disponíveis atualmente, de forma operacional nesse centro. Nesses experimentos um ou mais sistemas de observação são retidos no processo de assimilação de dados, a fim de se avaliar o impacto da exclusão de uma determinada observação na qualidade da previsão numérica de tempo. Assim, com base nos resultados obtidos é possível ter uma indicação da importância de cada sistema de observação no processo de assimilação/previsão de tempo utilizado nesse centro. A próxima seção descreve os experimentos realizados, assim como a metodologia de avaliação. Na seção 3, são apresentados os resultados relacionados ao desempenho do modelo, bem como os relacionados à análise de impacto. As conclusões são apresentadas na seção 4 .

\section{EXPERIMENTOS NUMÉRICOS E METODOLOGIA DE AVALIAÇÃO}

Experimentos de assimilação/previsão de tempo foram realizados no CPTEC utilizando o Global Physical-space Statistical Analysis System (GPSAS, Herdies et al., 2002), que representa uma combinação do Modelo Atmosférico de Circulação Geral do CPTEC (MGCA-CPTEC/COLA), em uma resolução T126 L28, e o esquema de assimilação de dados PSAS. Detalhes do MCGA-CPTEC/COLA e do sistema de assimilação de dados usados nesse estudo podem ser encontrados nos trabalhos de Cavalcanti et al. (2002) e Cohn et al. (1998), respectivamente.

Cada experimento foi definido conforme a seguinte nomenclatura:
CTR: inclui informações de altura geopotencial, pressão em superfície, umidade específica e vento zonal e meridional, oriundas de sistemas convencionais e distribuídas via Global Telecommunication System (GTS) e informações de vento, umidade e altura geopotencial inferidas por satélites, tais como: dados de vento por satélite (Cloud Track Wind - CTW), dados de vento em superfície sobre o oceano (QuikScat), água Precipitável (Total Precipitation water - TPW) estimadas a partir de informações do Special Sensor Microwave Imager (SSM/I), perfis verticais de temperatura e umidade inferidos pelo Advanced TIROS-N/NOAA Operational Vertical Sounder (ATOVS) e perfis verticais de altura geopotencial inferidos pelo sistema de sondagem AIRS/AMSU. Esse experimento é usado como referência (controle) para comparação dos resultados.

NOATOVS: é o controle menos os perfis atmosféricos inferidos pelo sistema ATOVS;

NOSR4: é o controle menos os dados convencionais e o vento estimado por satélite (CTW);

NOAIRS: é o controle menos os perfis de altura geopotencial inferidos pelo sistema de sondagem AIRS/AMSU;

NORET: é o controle menos todos os perfis de altura geopotencial dos sistemas ATOVS e AIRS/AMSU;

NOQSCAT: é o controle menos os dados de vento sobre o oceano (QuikScat);

NOCTW: é o controle menos os dados de vento estimados por satélite (CTW);

NOWIND: é o controle menos todos os dados de vento estimados por satélites (CTW e QuikScat);

NOTPW: é o controle menos os dados de TPW.

Esses experimentos foram realizados em um modo cíclico, no qual inicialmente, a partir de uma previsão de 6 horas (first-guess) e de observações disponíveis, o sistema de análise PSAS gera uma nova estimativa do estado da atmosfera num dado instante de tempo. Essa nova estimativa ou condição inicial servirá de base para a nova rodada do modelo e a partir de então se inicia o ciclo de previsões (Herdies et al., 2002). Previsões para até cinco (5) dias foram realizadas para o período de 01 a 31 de março de 2004. No entanto, as estatísticas aplicadas aos campos de análise e previsão, excluem os primeiros 5 (cinco) dias de cada experimento. Esta redução é feita com intuito de eliminar o impacto da remoção dos diferentes sistemas de observação nas primeiras rodadas do modelo e reduz o período de análise para 26 dias (6 a 31 de março).

Para se ter uma idéia da qualidade dos campos de análises gerados pelo GPSAS, os mesmos são comparados com as análises do NCEP. Neste caso, serão apresentados os coeficientes de correlação de anomalias e a raiz do erro médio quadrático entre os campos de análises do GPSAS e do NCEP. As análises do NCEP são utilizadas para verificação pelo fato dessas serem utilizadas paralelamente, 
para inicialização operacional do Modelo Global do CPTEC. A performance do modelo de previsão tem sido, por muito tempo (e permanece em centros operacionais), uma medida crucial do desempenho para um sistema de previsão de tempo. A ferramenta escolhida para avaliar essa performance é a correlação da anomalia, que é a correlação entre uma anomalia (isto é, diferença em relação à climatologia) de um campo da previsão e uma anomalia de um campo da análise da verificação. Usualmente, quando se avalia a inclusão de novas observações no sistema de assimilação de dados/previsão de tempo, cada experimento (com ou sem a inclusão de novas observações) é avaliado de encontro às suas próprias análises. Contudo, no contexto de estudos de impacto, uma representação mais precisa de cada tipo de observação é obtida quando a estimativa do estado da atmosfera, que melhor represente o campo observado, é usada para a avaliação. Neste estudo, utiliza-se o campo de análise do experimento CTR como referência. No entanto, vale salientar que os resultados das análises são robustos em relação à escolha da análise de referência. Testes foram realizados utilizando as análises do NCEP como referência e os resultados são similares para todas as regiões. Os principais campos examinados por esta verificação são a altura geopotencial em $500 \mathrm{hPa}$ e a pressão ao nível do mar. As correlações da anomalia da altura geopotencial em $500 \mathrm{hPa}$ e da pressão ao nível do mar foram calculadas na mesma grade do modelo, usando a análise do experimento de controle como a referência. A climatologia usada para esses cálculos corresponde a uma média de 48 anos (1950-1998), obtida da reanálise do NCEP. As regiões avaliadas são: $\mathrm{HN}$ entre $20^{\circ} \mathrm{N}-80^{\circ} \mathrm{N}$, HS entre $80^{\circ} \mathrm{S}-20^{\circ} \mathrm{S}$, região tropical entre $20^{\circ} \mathrm{S}-20^{\circ} \mathrm{N}$ e América do Sul $\left(50^{\circ} \mathrm{S}-10^{\circ} \mathrm{N}, 80^{\circ} \mathrm{W}-30^{\circ} \mathrm{W}\right)$. Os valores para a correlação de anomalias estão compreendidos entre -1 e 1, sendo o valor 1 relacionado a uma previsão perfeita. Geralmente, um valor maior que 0,6 é considerado como uma indicação de uma previsão útil (Krishnamurti et al., 2003).

Cálculos da raiz do erro médio quadrático das previsões em relação à análise do experimento de controle, e em relação aos dados de radiossondas somente sobre a região da América do Sul são também apresentados. No caso, da avaliação em relação aos dados de radiossondas, os campos do modelo foram interpolados para o mesmo ponto de observação e as diferenças entre as previsões e as observações foram utilizadas.

\section{1 - Distribuições espacial e temporal das observações}

Como descrito anteriormente, o GPSAS assimila informações oriundas de diferentes sistemas de observações. Freqüentemente, esses dados possuem resolução espacial mais densa que a resolução espacial da grade do modelo (campo de análise). Assim, a fim de evitar custo computacional desnecessário, um procedimento de redução de dados é aplicado para alguns desses tipos de informação. Para grande parte dos sistemas de informação, uma diluição dos dados é feita para uma grade regular com definição de aproximadamente 1,8 graus em longitude por 1,4 graus em latitude. A seguir, é apresentado de forma resumida como essas informações são utilizadas no GPSAS. Maiores detalhes envolvendo especificações de estatísticas de erro e do controle de qualidade das observações são encontrados em Bloom et al., 2005.

Uma das informações utilizada no GPSAS é a pressão em superfície (reduzida ao nível médio do mar) obtida a partir de estações em superfície, que tem uma distribuição espacial irregular, e possui elevações inferiores a 1000m do nível do mar. Essas observações são convertidas para altura geopotencial no nível de pressão, correspondente a uma estimativa "background" da pressão em superfície. Esta conversão da observação é obtida usando a relação hidrostática em conjunto com a suposição envolvendo um "lapse rate" sintético para pontos de grade acima do nível do mar, e estimativas de temperaturas da superfície obtidas a partir do "first-guess". Além de informações de estação de superfície, o GPSAS assimila informações de pressão e vento coletados por bóias, navios e plataformas oceânicas. Novamente, essas informações de pressão são também convertidas em altura geopotencial para as análises de ar superior. As observações de vento sobre o oceano são modificadas antes de serem assimiladas para representar vetores de ventos médios por camada. Uma fonte adicional de vento em superfície que á assimilada pelo GPSAS refere-se às informações obtidas pelo sensor QuikScat. Uma descrição desses dados pode ser encontrada em Leidner et al., 2000.

A fonte predominante de dados convencionais em ar superior para o GPSAS é a radiossondagem, que fornece informações de geopotencial, temperatura, vento e umidade. No entanto, o GPSAS assimila dados de altura geopotencial (não de temperatura), umidade e componentes do vento somente para níveis isobáricos padrões. A distribuição espacial desses dados é muito irregular, favorecendo áreas continentais do HN. Informações adicionais dessas variáveis são obtidas a partir de aeronaves e balões piloto.

Outros tipos de observação utilizados pelo GPSAS são os não convencionais obtidos a partir de informações de satélites. Essas informações apresentam uma distribuição espacial mais uniforme do que aquelas dos dados convencionais. Nesse estudo, o GPSAS utilizou três tipos de dados de satélites: os perfis de geopotencial gerados a partir dos sistemas de sondagem ATOVS e AIRS/AMSU, o vento estimado a partir do deslocamento de nuvens e o total de água precipitável integrada numa coluna (TPW). As informações utilizadas pelo sistema ATOVS e AIRS/AMSU são oriundas dos satélites de órbita polar NOAA e AQUA, respectivamente, e tipicamente, uma órbita desses 
satélites cobre diferentes regiões do globo. As informações de vento inferidas por satélite geoestacionário possuem uma cobertura espacial quase global, limitada entre as latitudes de $60^{\circ} \mathrm{S}-60^{\circ} \mathrm{N}$. Somente as informações de vento definidas nas camadas entre a superfície e $700 \mathrm{hPa}$ e acima de $700 \mathrm{hPa}$ foram usadas no GPSAS. No caso de TPW, as informações são obtidas a partir do sensor SSM/I (Ledvina e Pfaendtner, 1995), que opera a bordo do satélite de órbita polar do DMSP (Defense Meteorological Satellite Program), e foram inferidas pela aplicação do algoritmo de Wentz (Wentz et al, 1986). Para a assimilação da TPW, é realizada uma análise independente das outras observações, a fim de explorar a sua relação direta com o perfil de umidade específica do first-guess do modelo. O perfil de umidade específica resultante dessa análise é então assimilado pelo GPSAS. Nos experimentos realizados nesse estudo, essa variável foi assimilada somente sobre regiões oceânicas.

A Figura 1 ilustra a evolução temporal do número de observações aceitas, as quais são integradas para todos os níveis disponíveis, para cada tipo de observação descrita anteriormente e utilizada no experimento CTR. O número de observações está discriminado em informações de altura geopotencial, vento, água precipitável e umidade específica. A quantidade correspondente às informações de vento refere-se somente a uma componente. As oscilações na quantidade de observações de vento (CTW) são decorrentes da diminuição no número total de dados disponíveis em certos dias. É evidente que a maior parte das informações utilizadas por sistemas de assimilação nos diversos centros operacionais e, por conseguinte, pelo GPSAS é oriunda de satélites. Como mencionado anteriormente, os dados convencionais (Z-Conv, U, V-Conv e UMES) apresentam uma cobertura satisfatória somente em áreas continentais do HN (América do Norte, Europa e sudoeste da Ásia). Por outro lado, os dados de satélite (AIRS, ATOVS, TPW, QSCAT, CTW) possuem uma cobertura global. Sobre o HN, essas observações sobrepõem àquelas relacionadas aos dados convencionais e devido a sua qualidade inferior estas terão uma importância menor no processo de assimilação de dados. Sobre o HS e regiões tropicais, as sondagens remotas atuam como fonte predominante de informações na previsão de tempo operacional do CPTEC, principalmente em regiões oceânicas onde a disponibilidade de dados convencionais é baixa. Além disso, nota-se um maior número de informações, inferidas por satélites, associadas ao campo de massa (geopotencial) em relação às informações de vento e umidade. As evoluções temporais do número de informações aceitas pelo sistema GPSAS para cada experimento realizado são apresentadas na Figura 2. De forma geral, verifica-se que a exclusão de um determinado tipo de observação não afeta significativamente

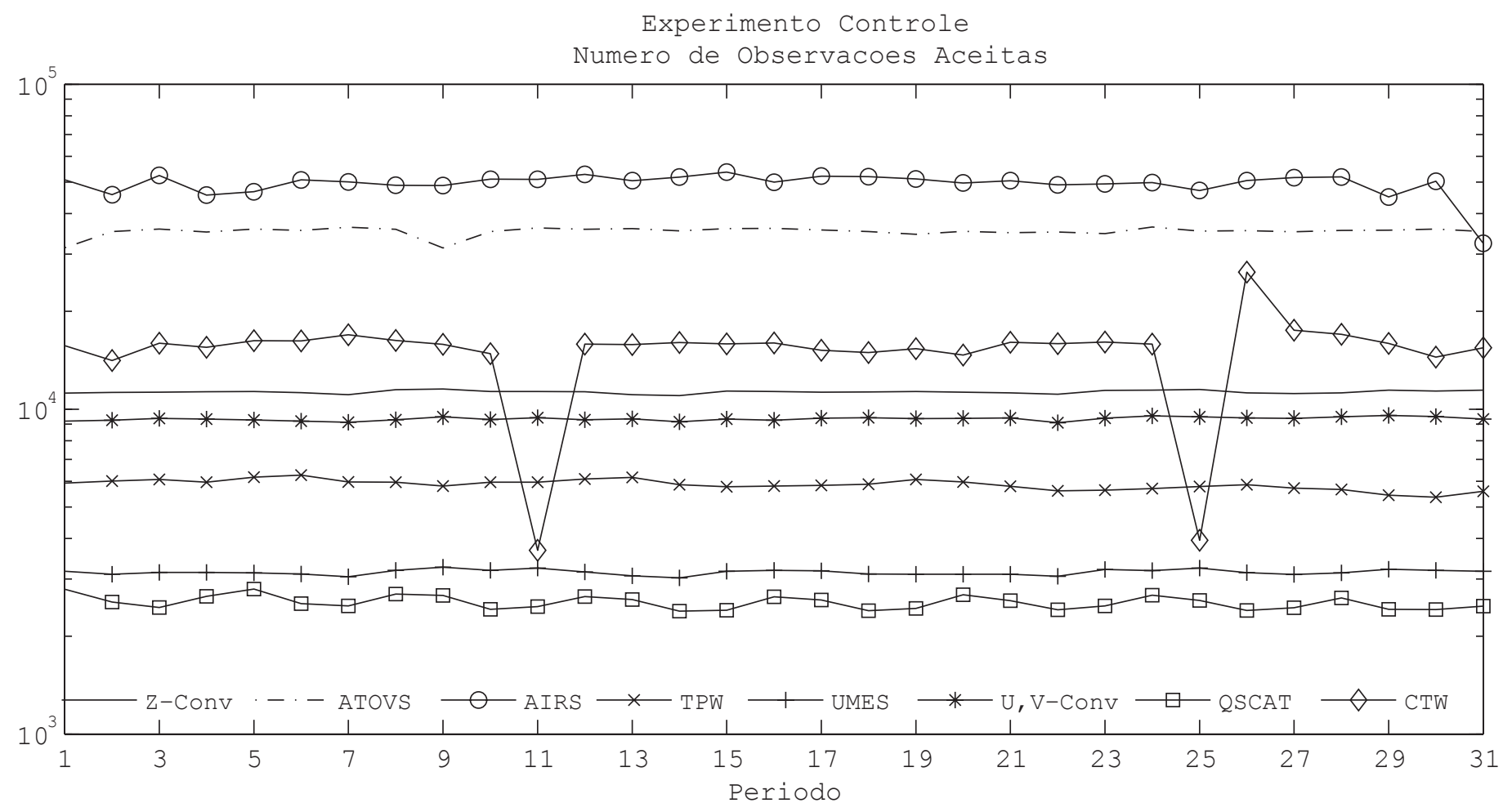

Figura 1 - Número total de observações de geopotencial (Z-Conv, ATOVS, AIRS), água precipitável (TPW), umidade específica (UMES) evento (U,V-Conv, QSCAT, CTW), assimiladas pelo GPSAS em função do tempo no horário das 12 UTC. Esses valores correspondem a valores integrados para todos os níveis da observação e ao experimento CTR. 
o número total de informações referentes aos outros sistemas, aceitas pelo GPSAS.

\section{RESULTADOS}

\section{1 - Qualidade das Análises Geradas pelo GPSAS}

Inicialmente avalia-se a qualidade das análises geradas pelo sistema GPSAS para os diversos experimentos realizados. Essa avaliação é realizada considerando o coeficiente de correlação de anomalias e a raiz do erro médio quadrático obtidos a partir dos campos de geopotencial em $500 \mathrm{hPa}$, pressão ao nível do mar, vento zonal em 850 e $250 \mathrm{hPa}$ e umidade específica em $850 \mathrm{hPa}$ gerados pelo sistema GPSAS e àqueles gerados pelo NCEP. Esta análise foi realizada considerando o período de 6 a 31 de março e o horário das 12 UTC.

A Figura 3 apresenta a evolução temporal dos coeficientes de correlação de anomalias para o campo de análise do geopotencial em $500 \mathrm{hPa}$ para as regiões do HN, HS, tropical e América do Sul, e experimentos CTR, NOSR4, NORET, NOWIND e NOTPW. Esses experimentos indicam que os dados convencionais, juntamente com estimativas de vento por satélite (CTW) são fontes primárias de informações sobre as regiões do HN, tropical e América do Sul. Por outro lado, sobre a região do
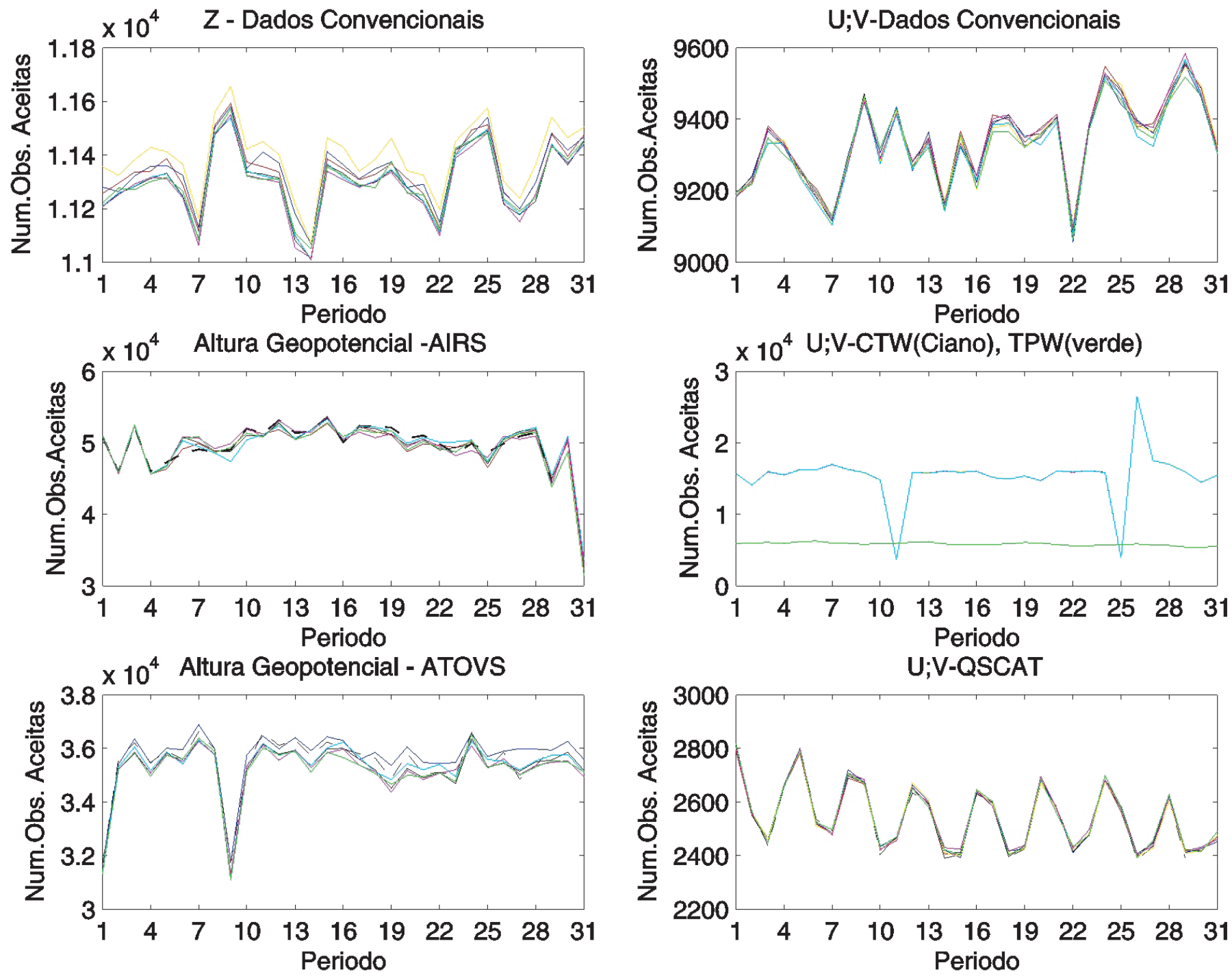

Figura 2- Número total de observações aceitas pelo sistema GPSAS em função do tempo, no horário das 12 UTC, para cada experimento realizado. As séries de observações são separadas em função da variável e da fonte de observação, para cada experimento realizado. Curva preta sólida corresponde ao experimento CTR, preta tracejada ao experimento NOSR4, azul ao experimento NOAIRS, vermelha ao experimento NOATOVS, amarela ao experimento NORET, ciano ao experimento NOQSCAT, magenta ao experimento NOTPW e finalmente a curva verde ao experimento NOCTW. 
HS, informações de satélite têm grande importância. Isso se deve ao fato da maior concentração de dados convencionais sobre regiões continentais do $\mathrm{HN}$ em relação ao HS, além da maior extensão de regiões oceânicas do HS sobre às do HN. Nota-se ainda, que a remoção dos dados de altura geopotencial oriundos de satélites (experimento NORET) teve um maior impacto no
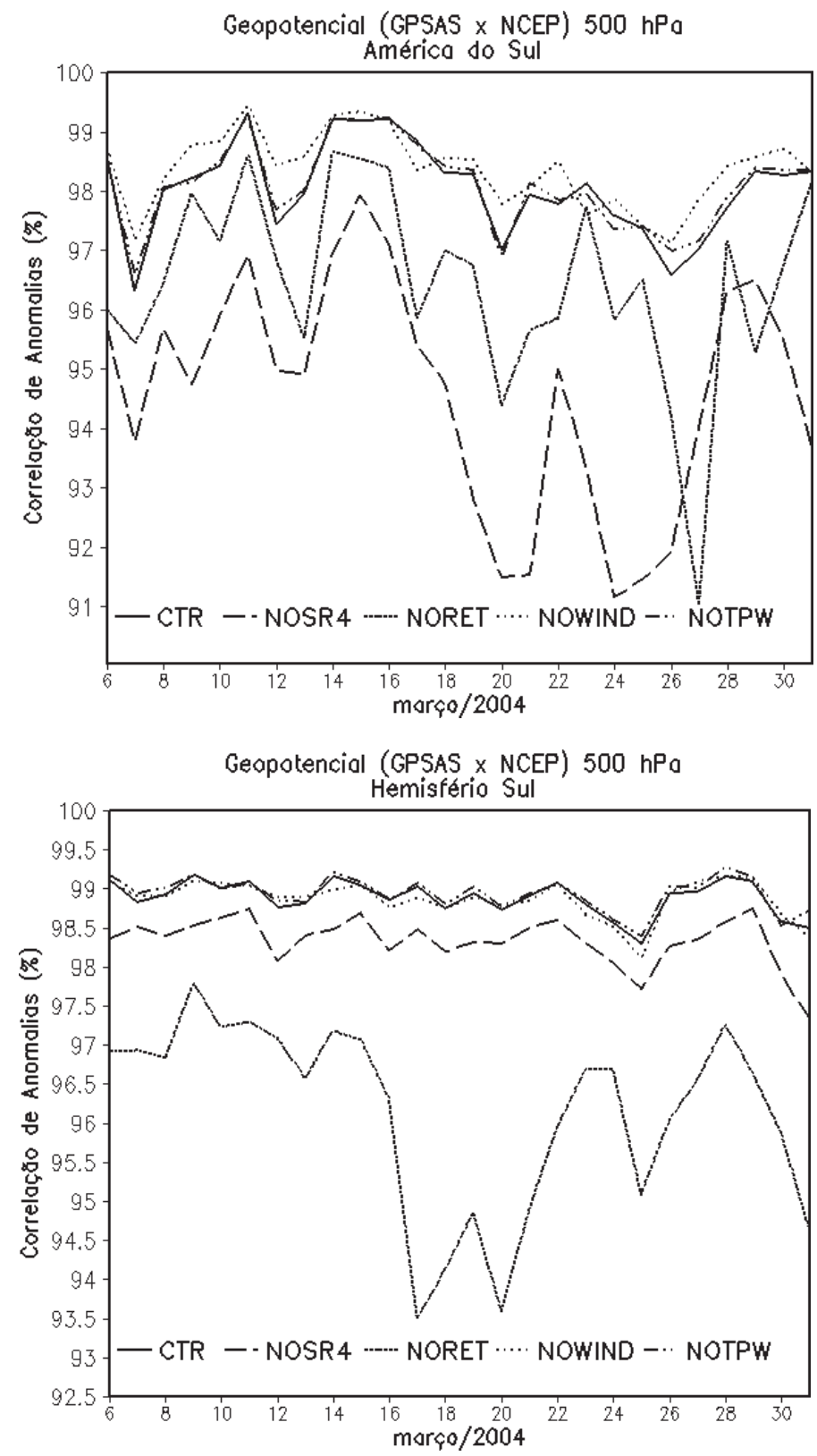

campo de análise em relação ao experimento com a remoção dos dados de vento inferidos por satélite (experimento NOWIND), para todas as áreas analisadas. A remoção dos dados de TPW apresentou resultado neutro.

Consistente com as análises de correlação, os valores de EMQ para os campos de geopotencial em 500 hPa e da pressão
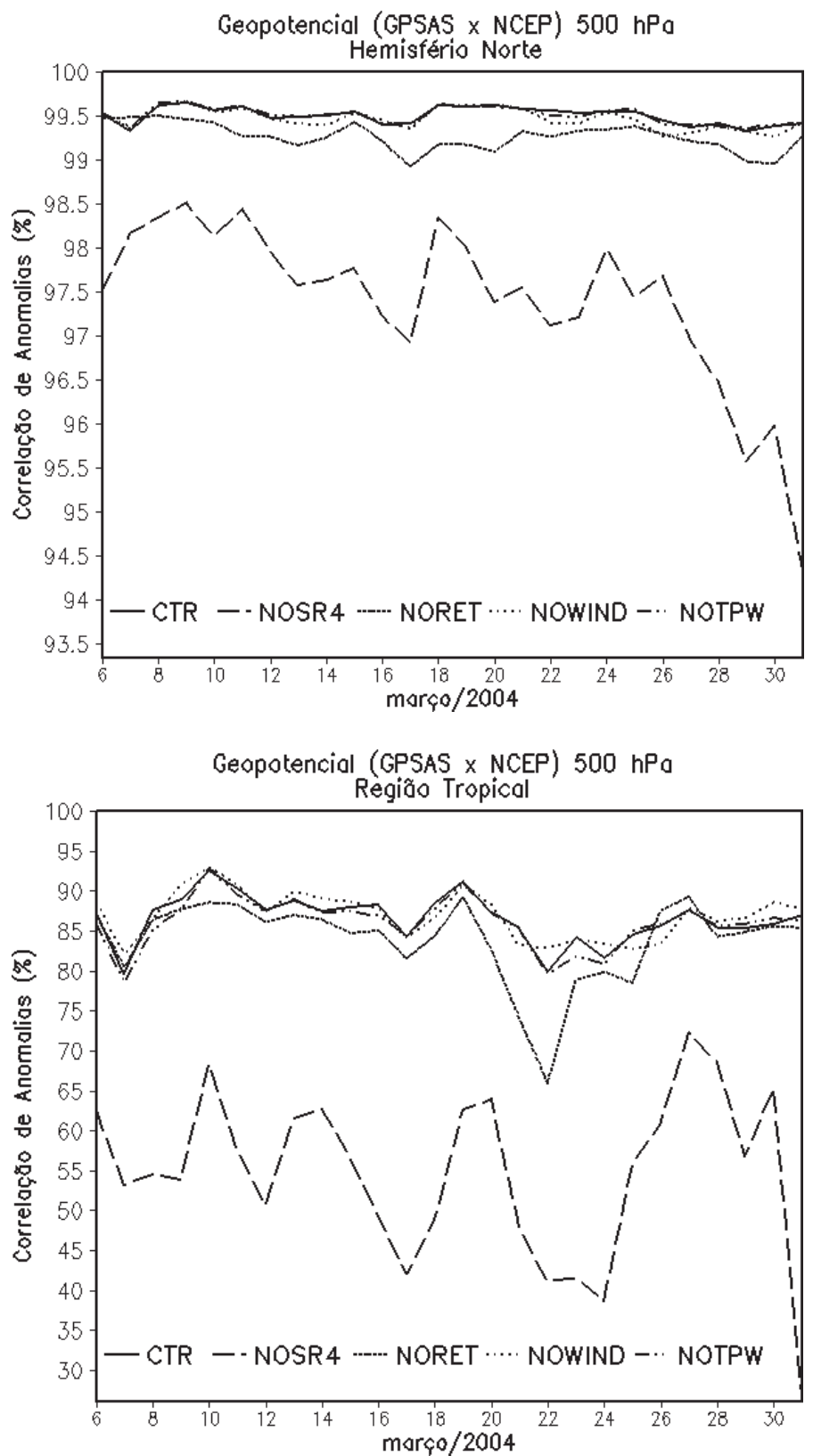

Figura 3 - Correlação de anomalias das análises do geopotencial em 500 hPa, geradas pelo GPSAS, usando os campos de análises do NCEP como referência, médio para as regiões do Hemisfério Norte, Hemisfério Sul, Tropical e América do Sul. Os resultados apresentados nesta figura referemse aos experimentos: CTR, NOSR4, NORET, NOWIND, NOTPW. 
ao nível do mar (PNM) para os campos de análises, médios para as regiões do HN e da América do Sul (figuras não apresentadas), indicam que os campos de análises obtidos são ligeiramente menos precisos quando as informações convencionais são excluídas da assimilação (NOSR4), comparadas àquelas obtidas com a inclusão desses dados (CTR). Sobre o HS, maiores valores de EMQ são obtidos quando as informações de geopotencial inferidas por satélites (NORET) são excluídas do processo de assimilação. Esse resultado está associado ao fato de existir nas regiões do HN e América do Sul uma maior concentração de dados de estações de superfície e radiossondagem. Máximos nas regiões oceânicas do HS, para o experimento NORET, refletem a escassez de informações convencionais nessas regiões.

A evolução temporal dos valores de EMQ para os campos da componente zonal do vento em $850 \mathrm{hPa}$ e $250 \mathrm{hPa}$, médios para as regiões do HN, HS, tropical e da América do Sul são
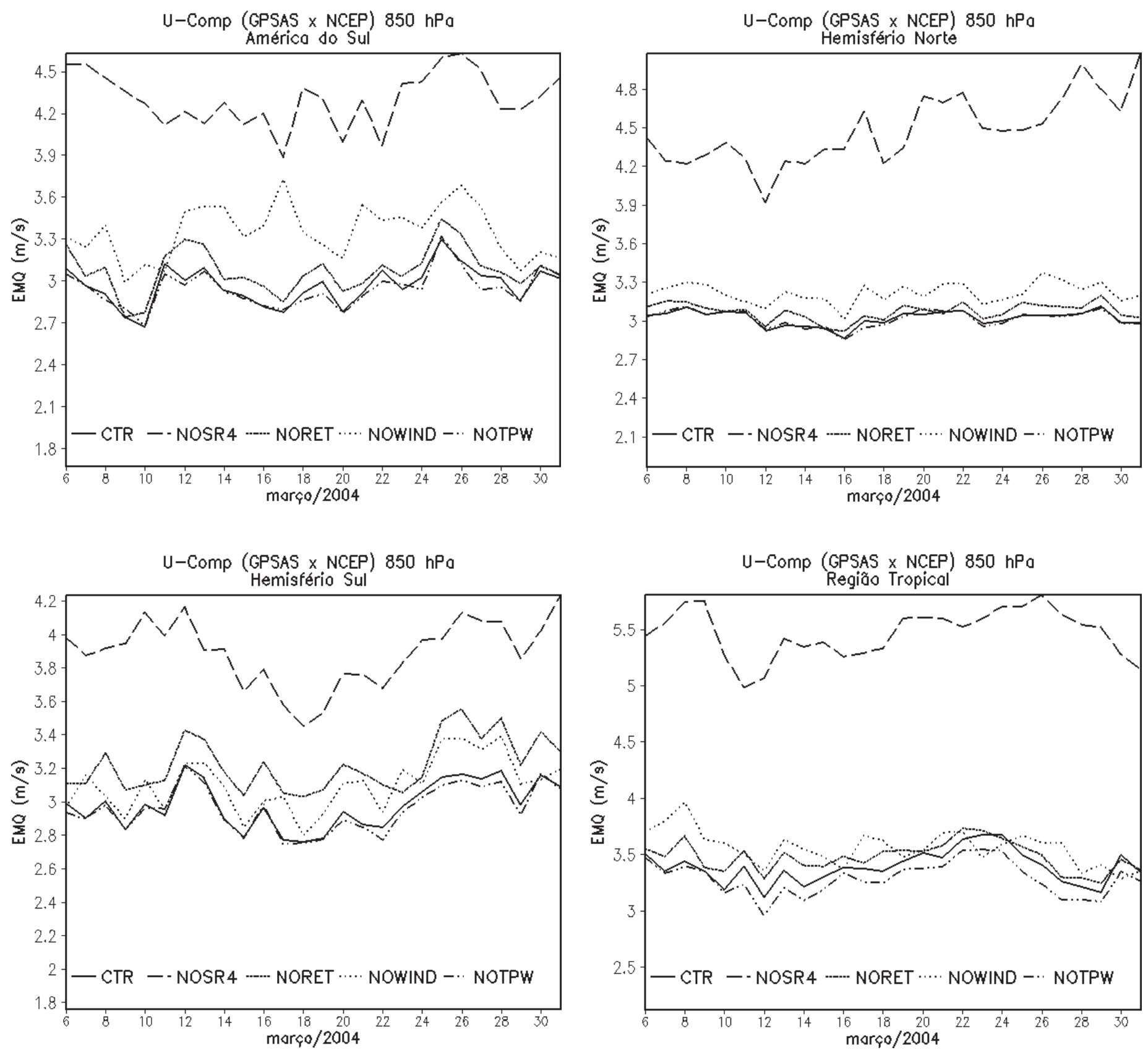

Figura 4 - Evolução temporal do erro médio quadrático das análises da componente zonal do vento em 850 hPa, geradas pelo GPSAS, médio para as regiões do Hemisfério Norte, Hemisfério Sul, Tropical e América do Sul. Os resultados apresentados nesta figura referem-se aos experimentos: CTR, NOSR4, NORET, NOWIND, NOTPW. Esses erros foram calculados utilizando as análises do NCEP como referência. 
apresentados nas Figuras 4 e 5, respectivamente. Exceto para a região do HS, os maiores erros mostrados nessas figuras ocorrem para o experimento NOSR4, seguido pelo experimento NOWIND. Para o HS, a remoção das informações de geopotencial tem um impacto maior do que a remoção de informações de vento, mesmo quando analisamos o campo de vento.

Alterações no campo de umidade específica em $850 \mathrm{hPa}$ à exclusão de algum do sistemas de observação são apresentadas na Figura 6. Nesta figura os maiores valores de EMQ ocorrem sobre as regiões tropical e da América do Sul. Nota-se ainda que o
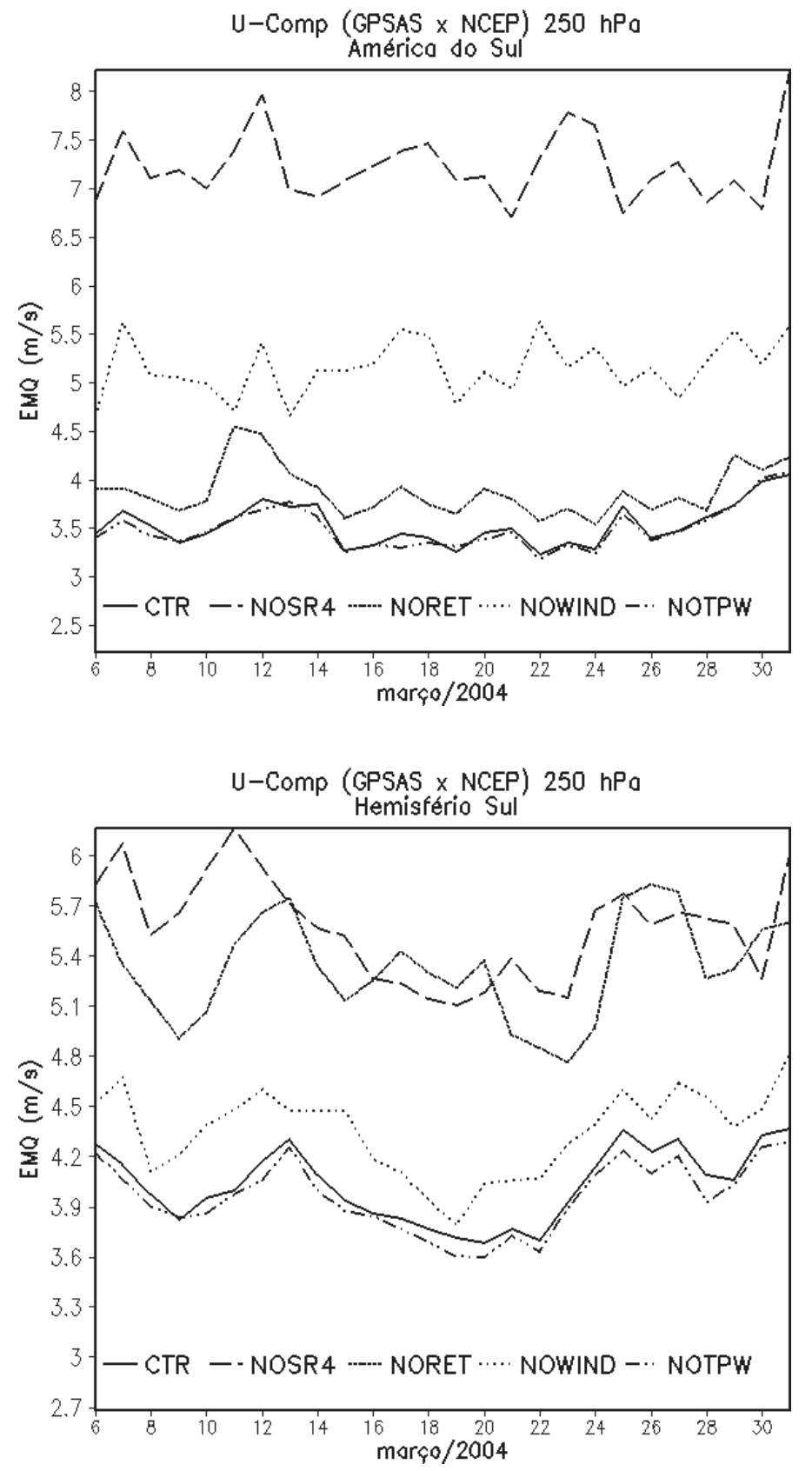

EMQ relativo ao experimento NOSR4 é maior, nas regiões do HN e HS, ou comparável aos EMQ relativo ao experimento NOTPW nas regiões tropical e da América do Sul. Isso decorre do fato que no experimento NOSR4 são excluídos os perfis verticais de umidade específica oriundos de radiossondagens. Desta forma, em regiões com maior disponibilidade de radiossondagens, como o caso do HN, esses dados dominam a análise. Por outro lado, nos trópicos, as informações de TPW dominam devido à pobre cobertura espacial dos dados convencionais.

Em adição aos resultados obtidos considerando os
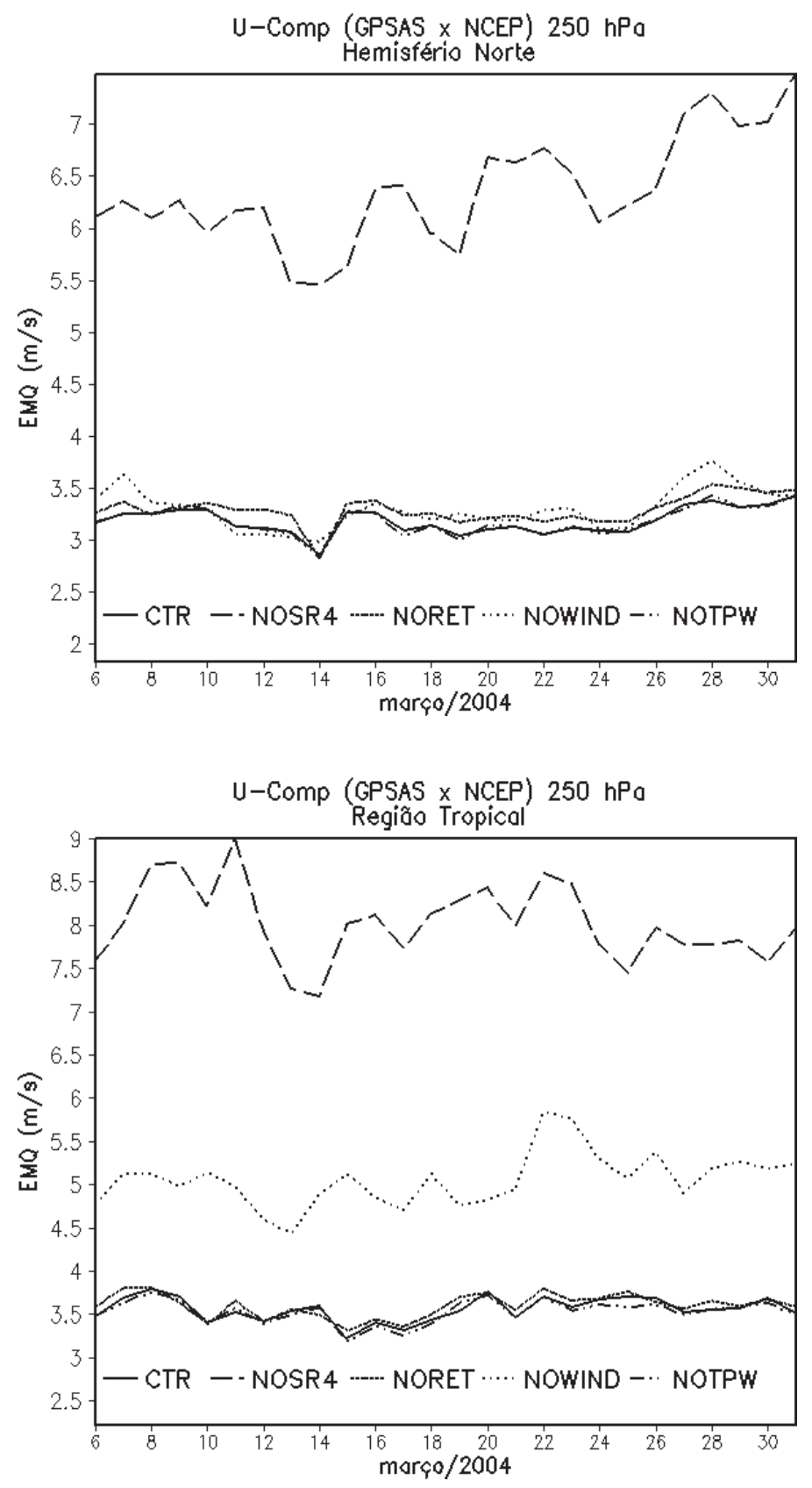

Figura 5 - Idem a Figura 4, exceto para a componente zonal do vento em $250 \mathrm{hPa}$. 
campos de análises do NCEP como referência, foram calculados os valores do EMQ, médios para a região da América do Sul, para os campos de análises para a altura geopotencial em 500 $\mathrm{hPa}$, para a componente zonal do vento em 850 e $250 \mathrm{hPa}$, e para a umidade específica em $850 \mathrm{hPa}$, obtidos em relação ao campo observado (radiossondas), considerando a condição inicial das 12 UTC (Figuras não mostradas). Esses cálculos reproduzem os resultados obtidos considerando as análises do NCEP como referência, exceto pelo fato que a magnitude
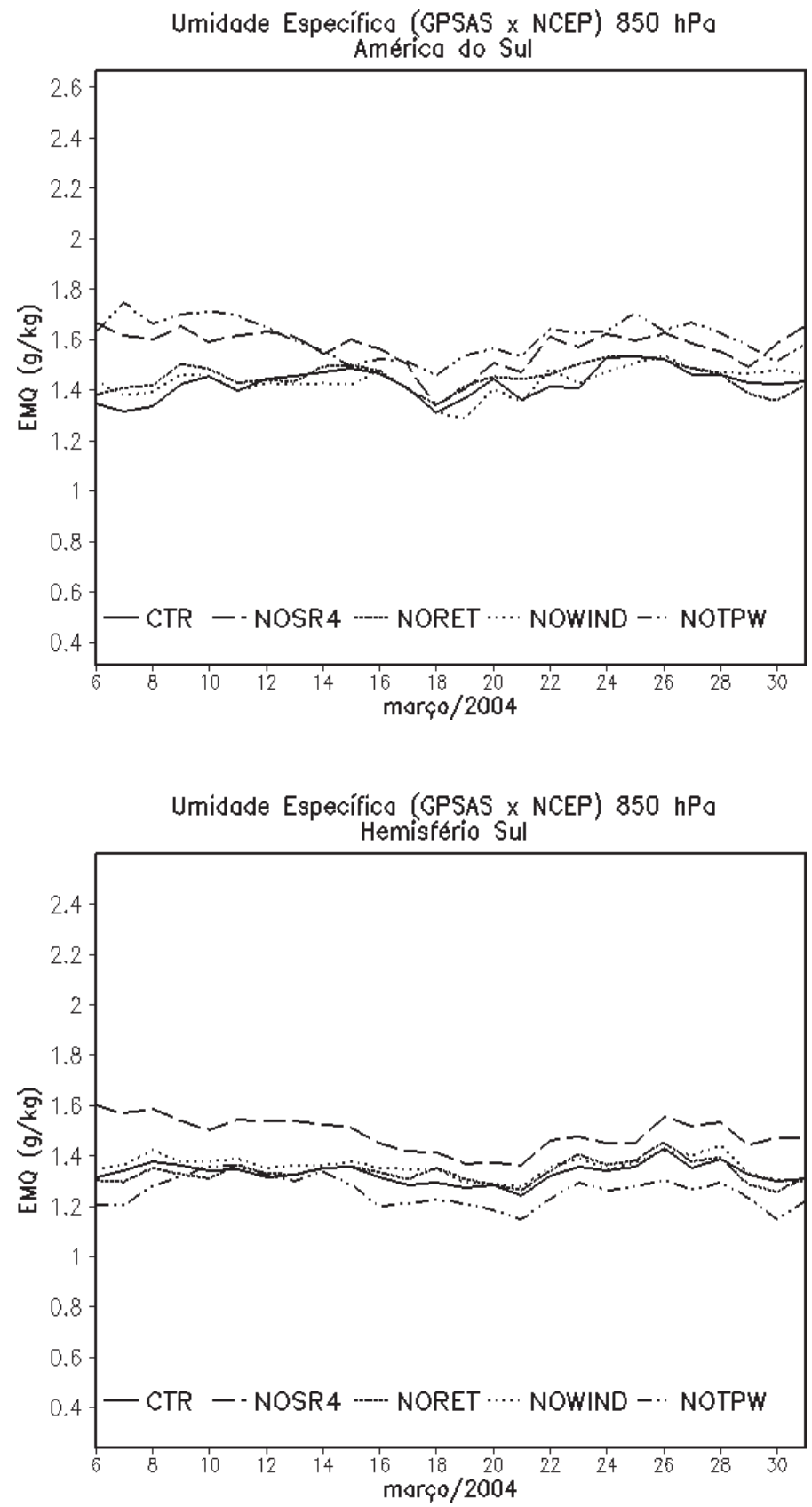

dos erros é menor. Essa redução do erro está associada ao fato que as radiossondagens utilizadas para a verificação foram anteriormente utilizadas no processo de assimilação.

\section{2 - Impacto nas Previsões}

A Figura 7 mostra os coeficientes de CA da altura geopotencial em $500 \mathrm{hPa}$ para o MCGA- CPTEC/COLA sobre as regiões do HN, HS, região tropical e da América do Sul,
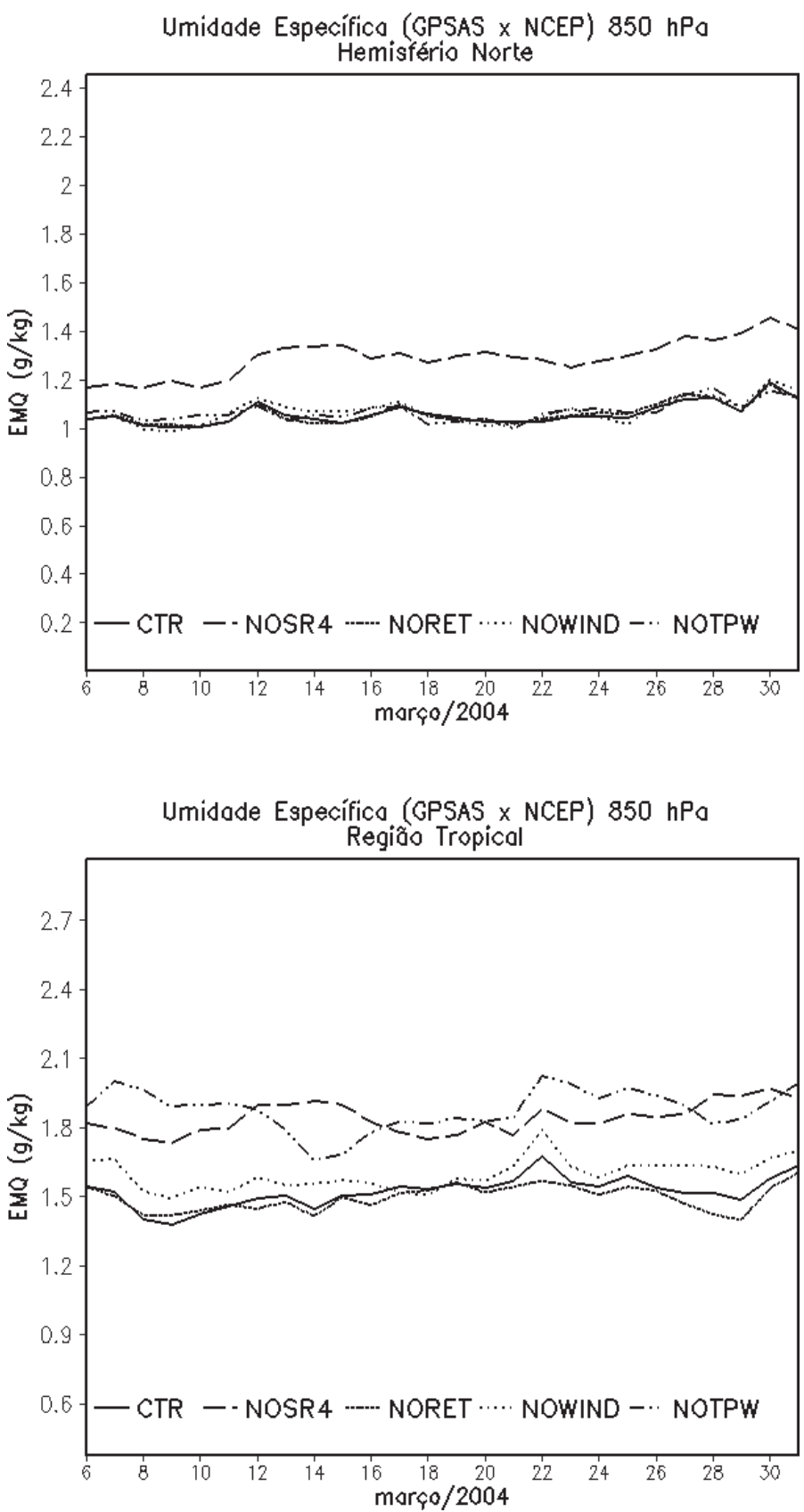

Figura 6 - Idem a Figura 4, exceto para a umidade específica em $850 \mathrm{hPa}$. 
respectivamente, para previsões de 1 a 5 dias, correspondentes a cinco dos nove experimentos realizados. São eles: os experimentos CTR, NORET, NOSR4, NOWIND e NOTPW. As correlações de anomalias representam uma média temporal dos impactos positivos/negativos. Resultados apresentados nessas figuras confirmam que o dado convencional é fonte primária de informação utilizada pelo GPSAS no HN, para esse mês analisado, devido a maior concentração de estações de superfície e radiossondagens nessa região. Esta conclusão se deve ao fato
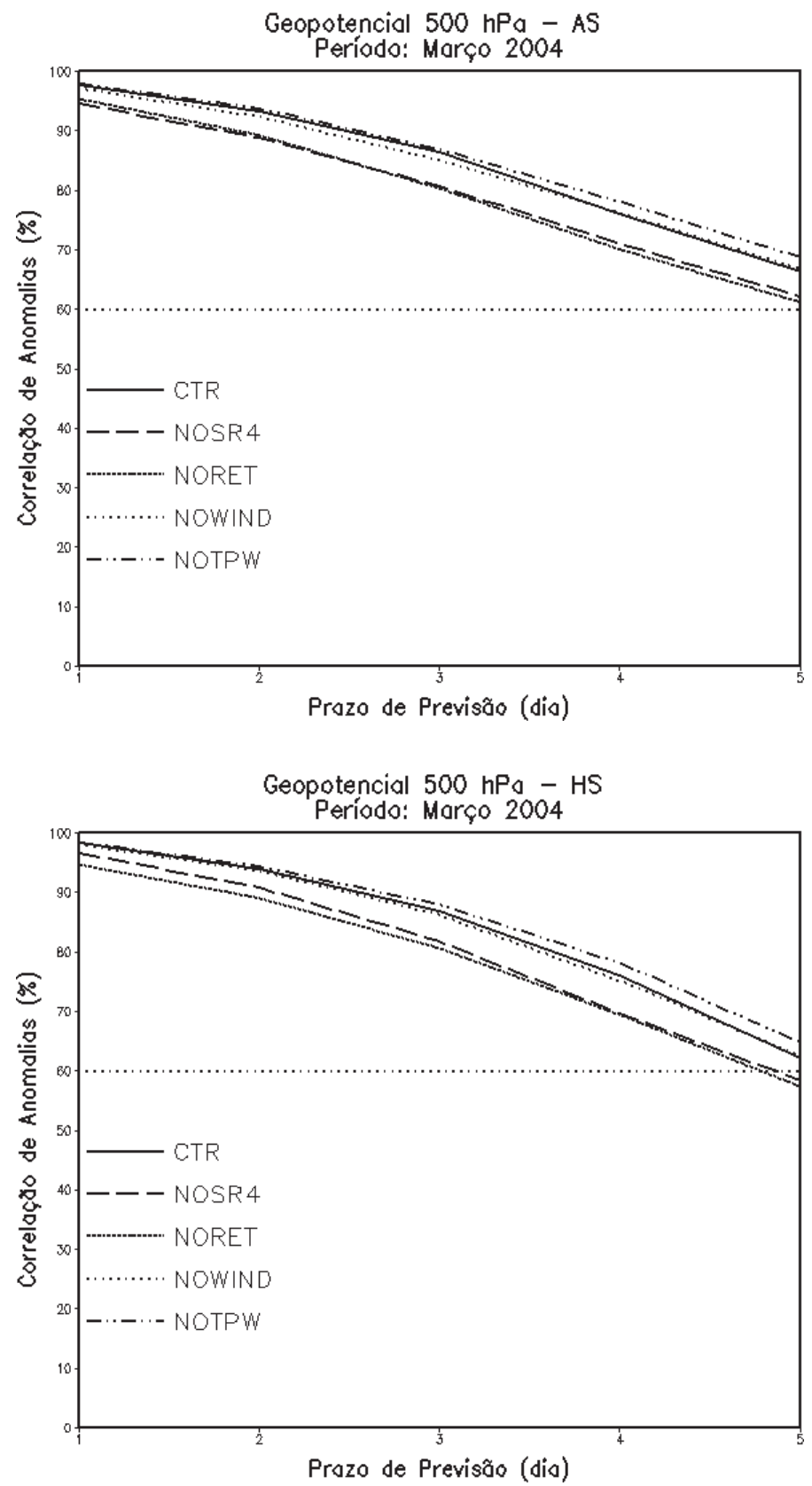

de que a retenção dos dados convencionais (NOSR4) fez com que a performance da previsão apresentasse uma maior redução em relação aos outros experimentos com dados retidos (NORET, NOWIND, NOTPW). Vale salientar, que o experimento NOSR4 exclui também os dados de vento estimados por satélite (CTW), no entanto experimentos complementares indicam que o impacto da exclusão desses dados para essa região é neutro. Por outro lado, a exclusão de perfis de geopotencial inferidos por satélites (NORET), gerou o maior impacto na
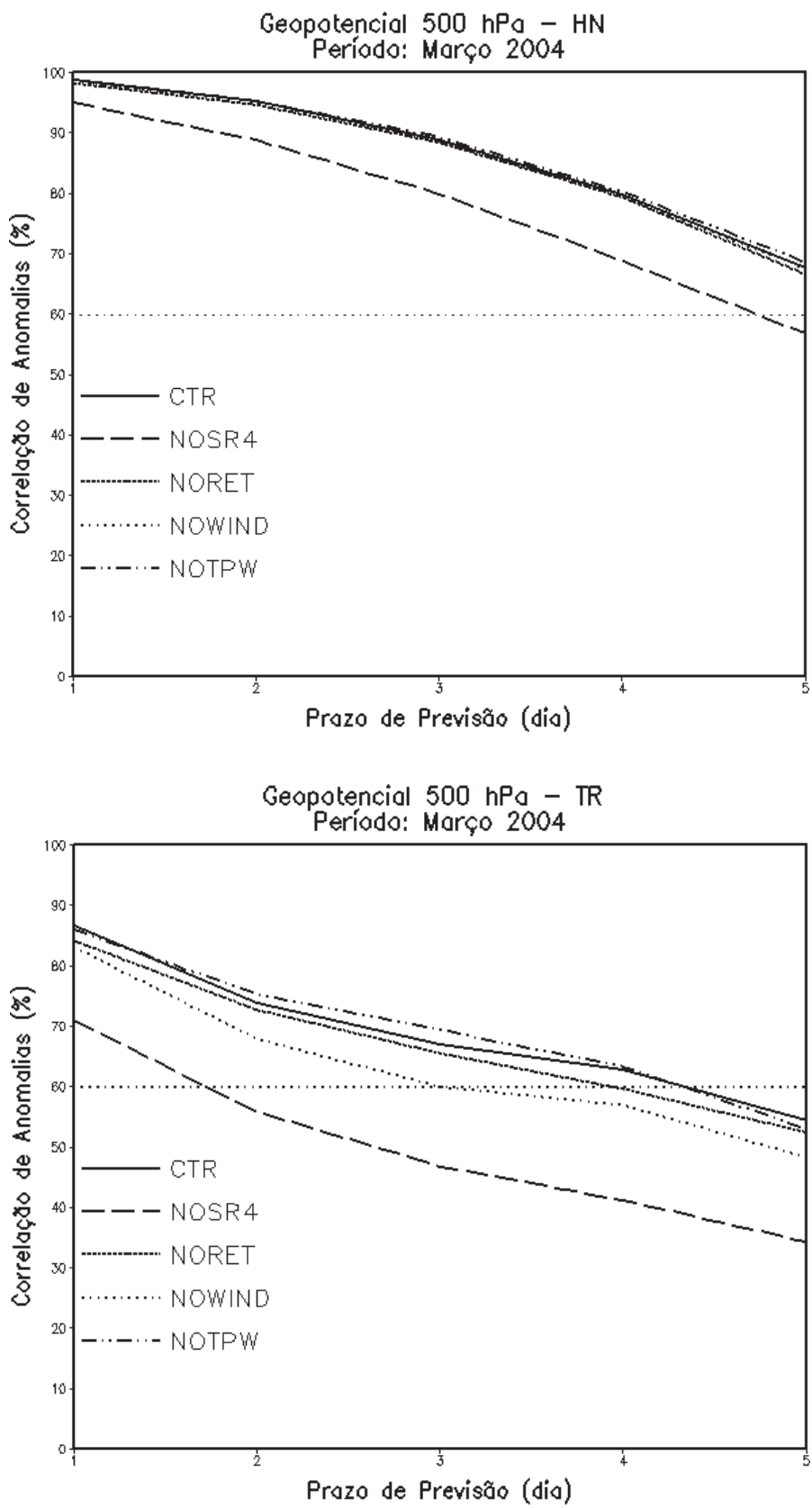

Figura 7 - Coeficiente de Correlação de Anomalias do geopotencial em 500 hPa para MCGA-CPTEC/COLA para os experimentos: CTR, NOSR4, NORET, NOWIND, NOTPW, para as regiões do Hemisfério Norte, Hemisfério Sul, Tropical e América do Sul. Esses resultados foram obtidos utilizando as análises do experimento CTR como referência. 
performance da previsão sobre o HS, seguida pela exclusão dos dados convencionais (NOSR4) e pela exclusão somente de informações de vento geradas por satélite (NOWIND). Sobre o HS, o impacto positivo na previsão de médio prazo, gerado pela inclusão das informações de geopotencial provenientes de satélites, foi de aproximadamente 6 horas para a previsão de cinco dias. Contrariamente, a exclusão dos dados de água precipitável parece ser favorável a uma melhora na performance da previsão sobre o HS. No entanto, como mostrado por Sapucci et al. (2006), a assimilação dessa variável é capaz de minimizar os erros do modelo em caracterizar a estrutura vertical da umidade, melhorando a previsibilidade das demais variáveis prognósticas do modelo nas camadas mais elevadas da troposfera. Comportamento similar ao que ocorre no HS é notado no desempenho da previsão sobre a região da América do Sul. Isso indica que a inclusão de perfis verticais de geopotencial
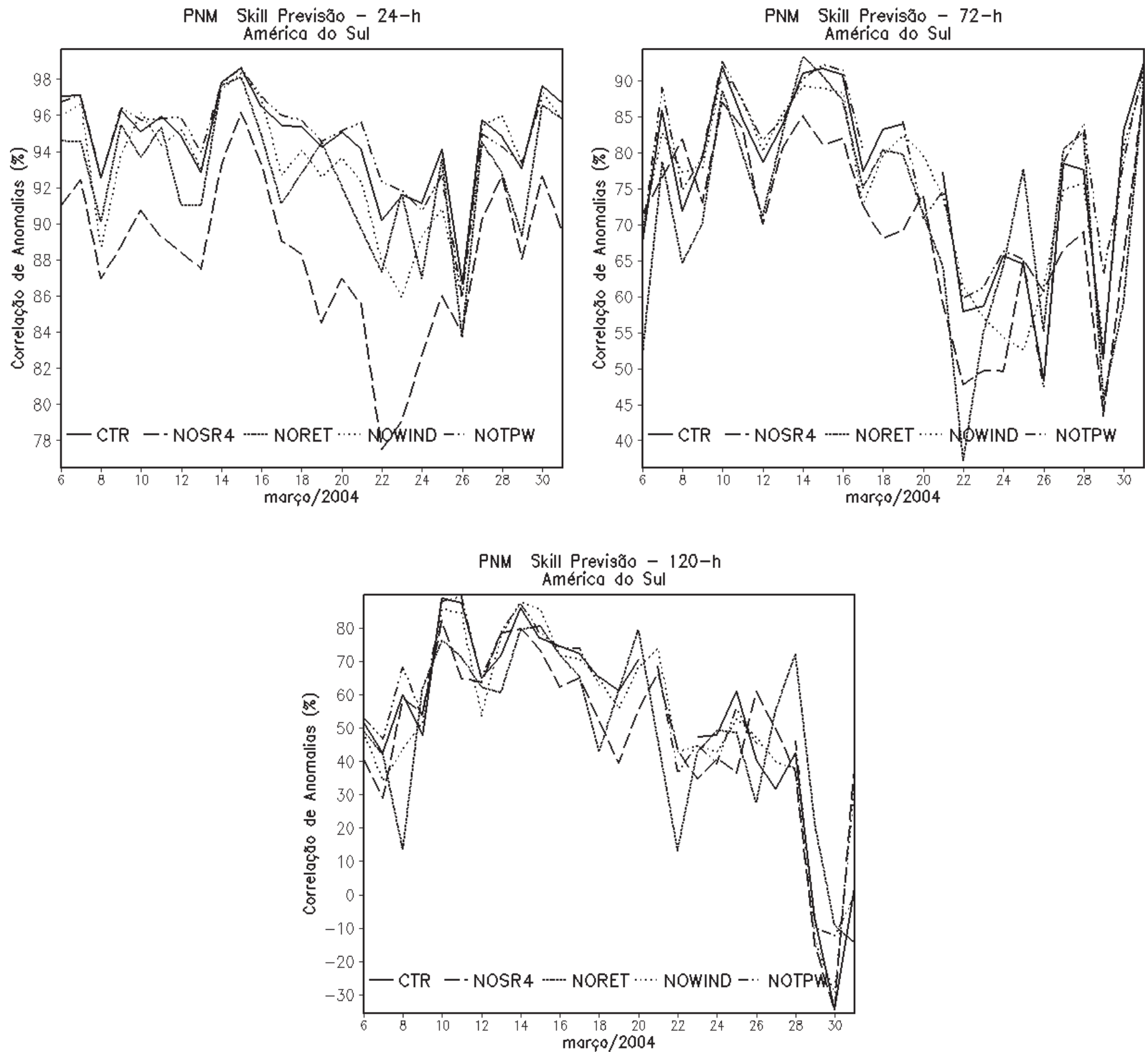

Figura 8 - Evolução temporal do Coeficiente de Correlação de Anomalias da PNM, médio sobre a região da América do Sul para os experimentos: CTR, NOSR4, NORET, NOWIND e NOTPW, para 24, 72 e 120 horas de previsão. Esses resultados foram obtidos utilizando as análises do experimento CTR como referência. 
inferidos por satélites melhora a qualidade das previsões, reduzindo a incerteza em regiões com poucas observações convencionais. Para a região tropical, a remoção dos dados de vento (NOWIND) tem um maior impacto em relação aos dados de geopotencial. Isto era de se esperar, uma vez que baseado na teoria do ajuste geostrófico, o vento tende a se ajustar aos campos de massa em altas latitudes, e vice versa em baixas latitudes. Conseqüentemente, a assimilação de campos de massa será mais

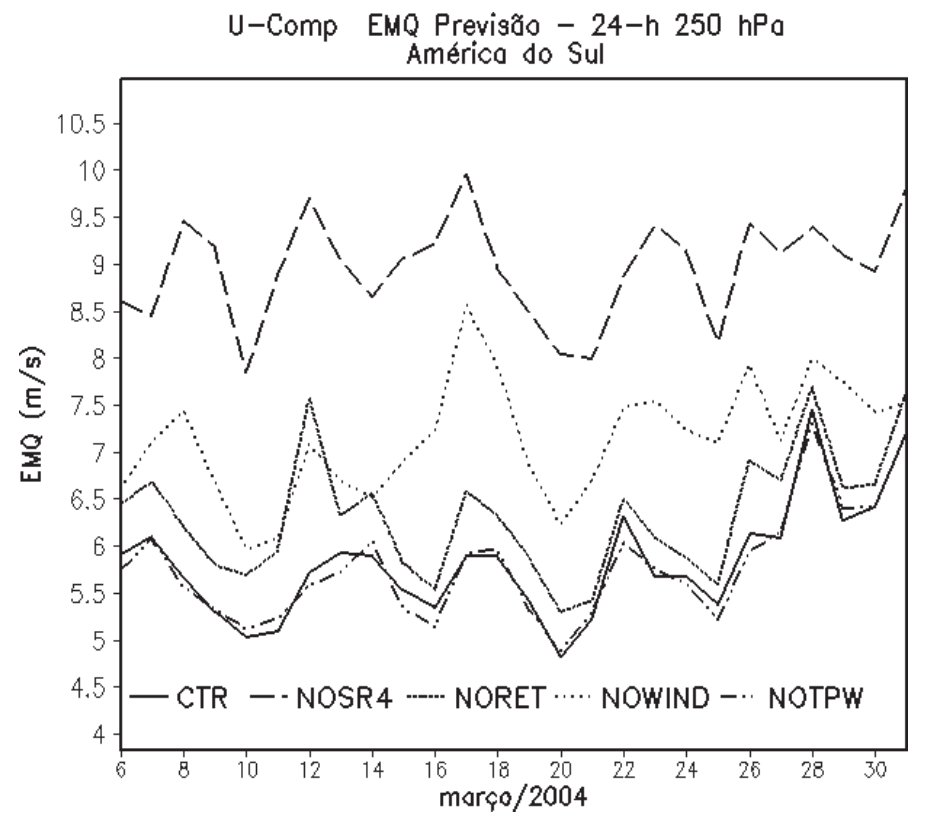

eficaz em altas latitudes, enquanto que o vento é uma variável crucial para determinar corretamente a dinâmica tropical.

A Figura 8 mostra a evolução diária do coeficiente de correlação de anomalias para pressão ao nível médio do mar, médios sobre a região da América do Sul, para os cinco experimentos apresentados na Figura 7 e para as previsões de 24, 72 e 120 horas. É evidente nestas figuras que, paralelamente, aos dados convencionais, que apresentam um maior impacto

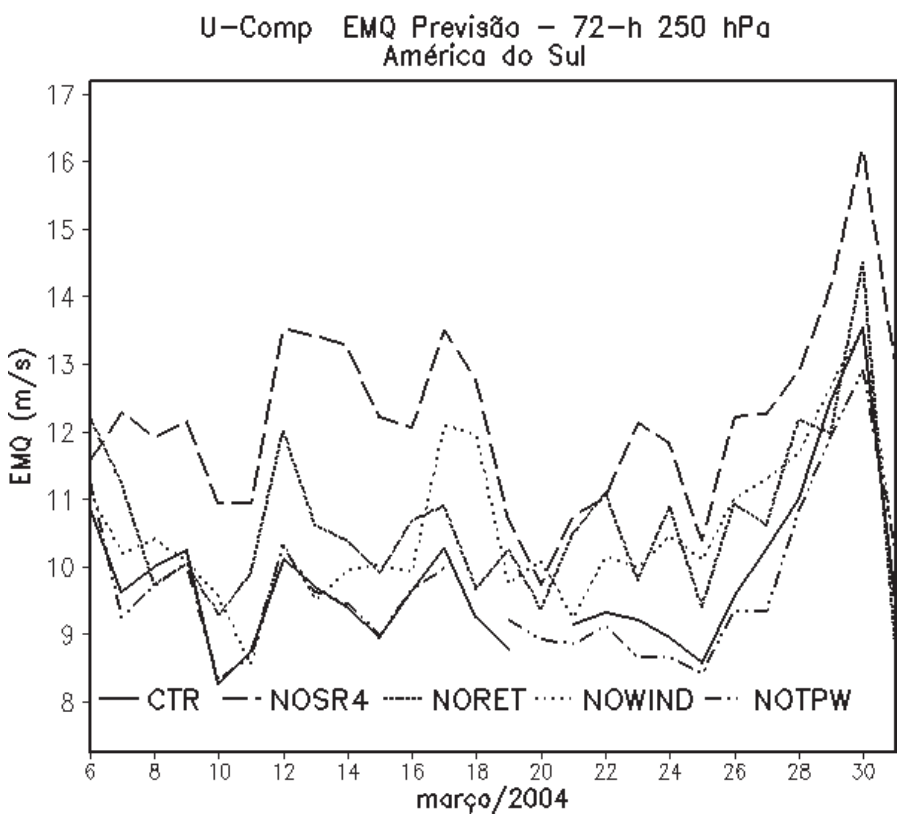

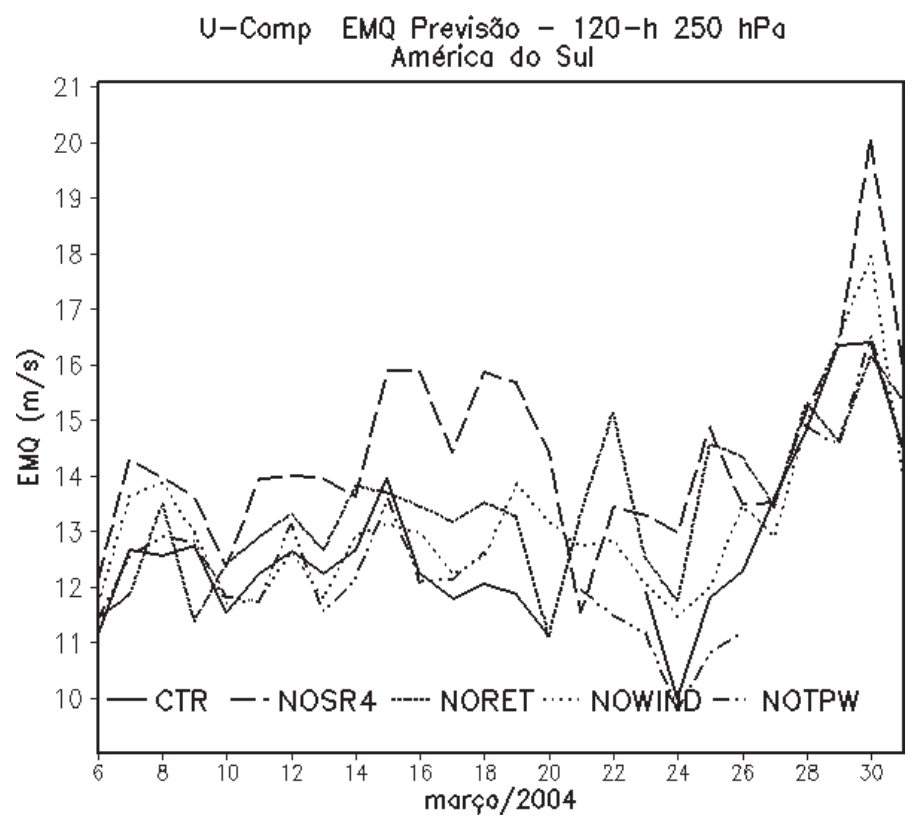

Figura 9 - Evolução temporal do EMQ da componente zonal do vento em $250 \mathrm{hPa}$, médio sobre a região da América do Sul para os experimentos: CTR, NOSR4, NORET, NOWIND e NOTPW, para 24, 72 e 120 horas de previsão. Esses resultados foram obtidos utilizando as análises do experimento CTR como referência. 
(experimento NOSR4), as outras informações de geopotencial e vento estimadas por satélites (NOWIND, NORET) mostram um impacto significativo na performance das previsões sobre a América do Sul. Outra característica notável nessas séries, é a baixa performance do modelo para essa região durante os últimos dez dias do mês, para previsões de 72 e 120 horas. Esse período coincide com o período de ocorrência do evento Catarina (McTaggart-Cowan, et al. 2006), no entanto, foge

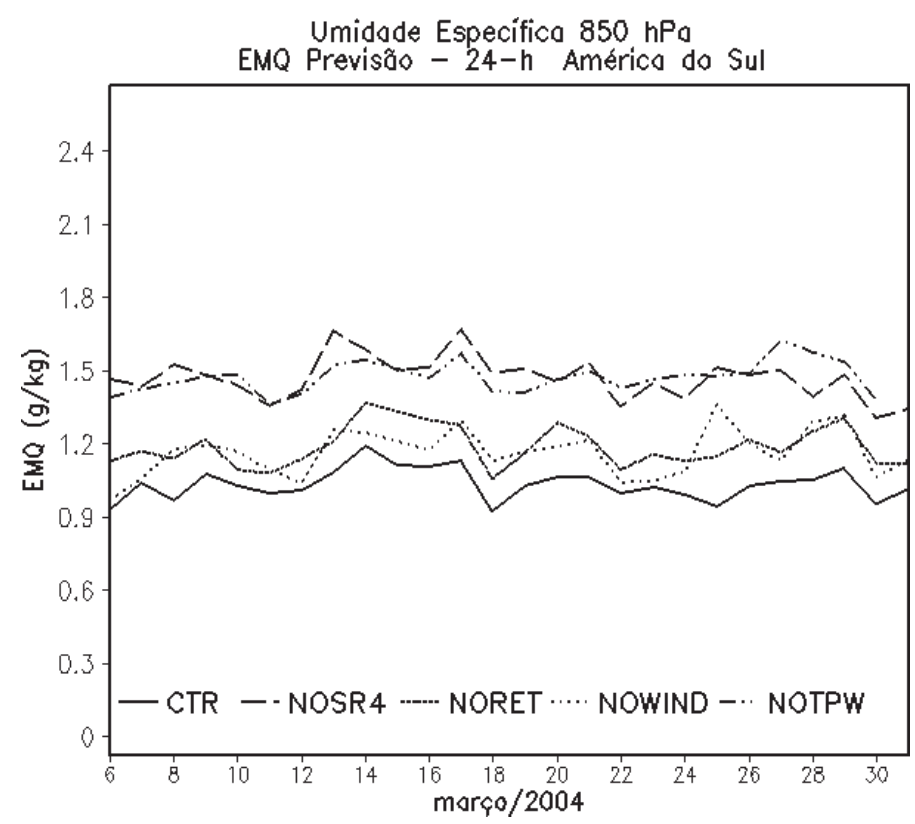

do escopo deste trabalho avaliar o impacto das diversas fontes de observação na definição de eventos extremos dessa natureza. Um trabalho complementar está sendo realizado com o objetivo de avaliar o impacto dos diferentes tipos de observação no desempenho do modelo, principalmente, em relação a posição, intensidade e estrutura dos ciclones

As evoluções diárias dos valores de EMQ para os campos da componente zonal do vento em $250 \mathrm{hPa}$, médios

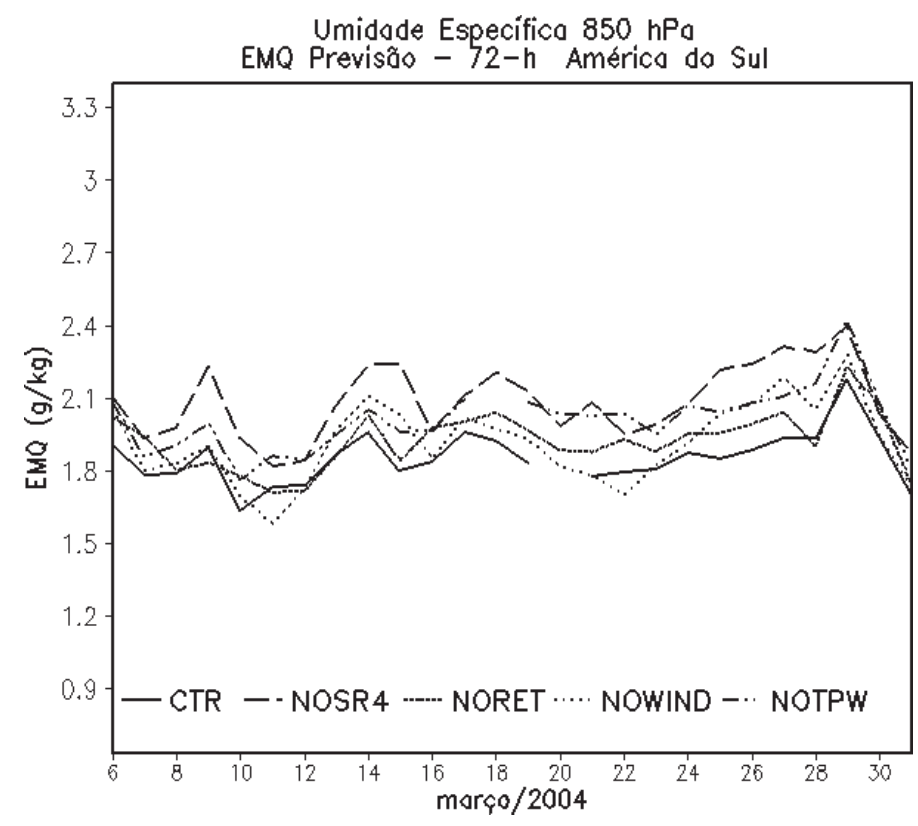

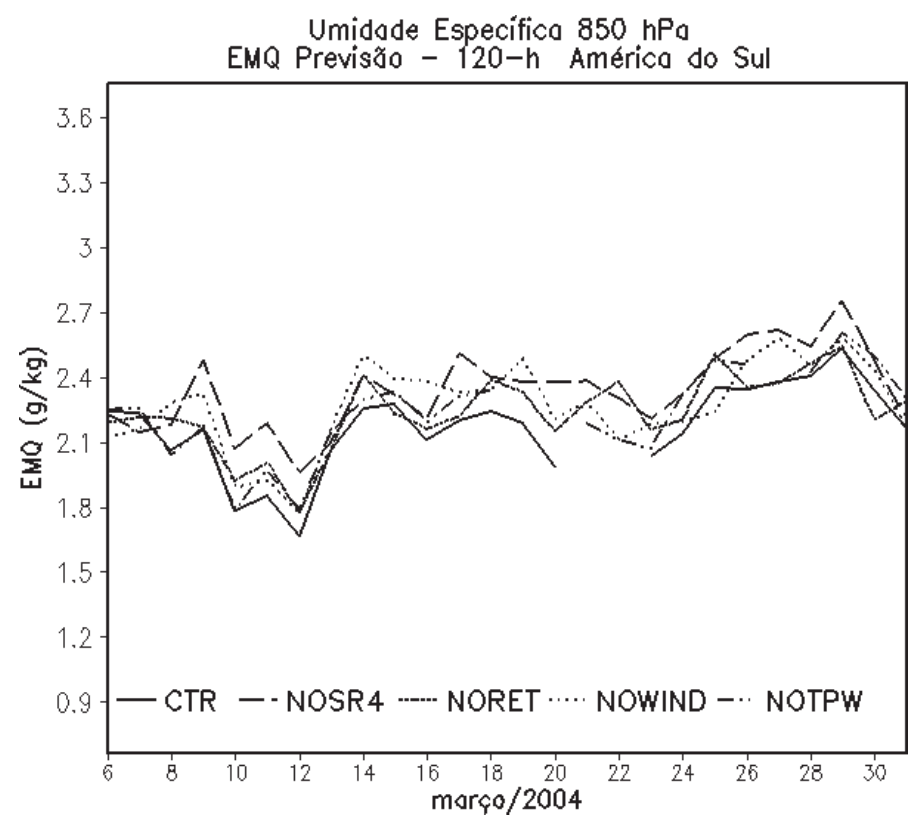

Figura 10 - Evolução temporal do EMQ da umidade específica em $850 \mathrm{hPa}$, médio sobre a região da América do Sul para os experimentos CTR, NOSR4, NORET, NOWIND e NOTPW, para 24, 72 e 120 horas de previsão. Esses resultados foram obtidos utilizando as análises do experimento CTR como referência. 
sobre a região da América do Sul, para os cinco experimentos apresentados na Figura 7 e para as previsões de 24, 72 e 120 horas são apresentadas na Figura 9. É observado nessa figura que o maior impacto na previsão do vento zonal em $250 \mathrm{hPa}$, ocorre quando os dados convencionais juntamente com as informações de vento estimadas por satélite (experimentos NOSR4 e NOWIND) são excluídos do processo de assimilação. Nota-se ainda que as estimativas de vento por satélite produzem
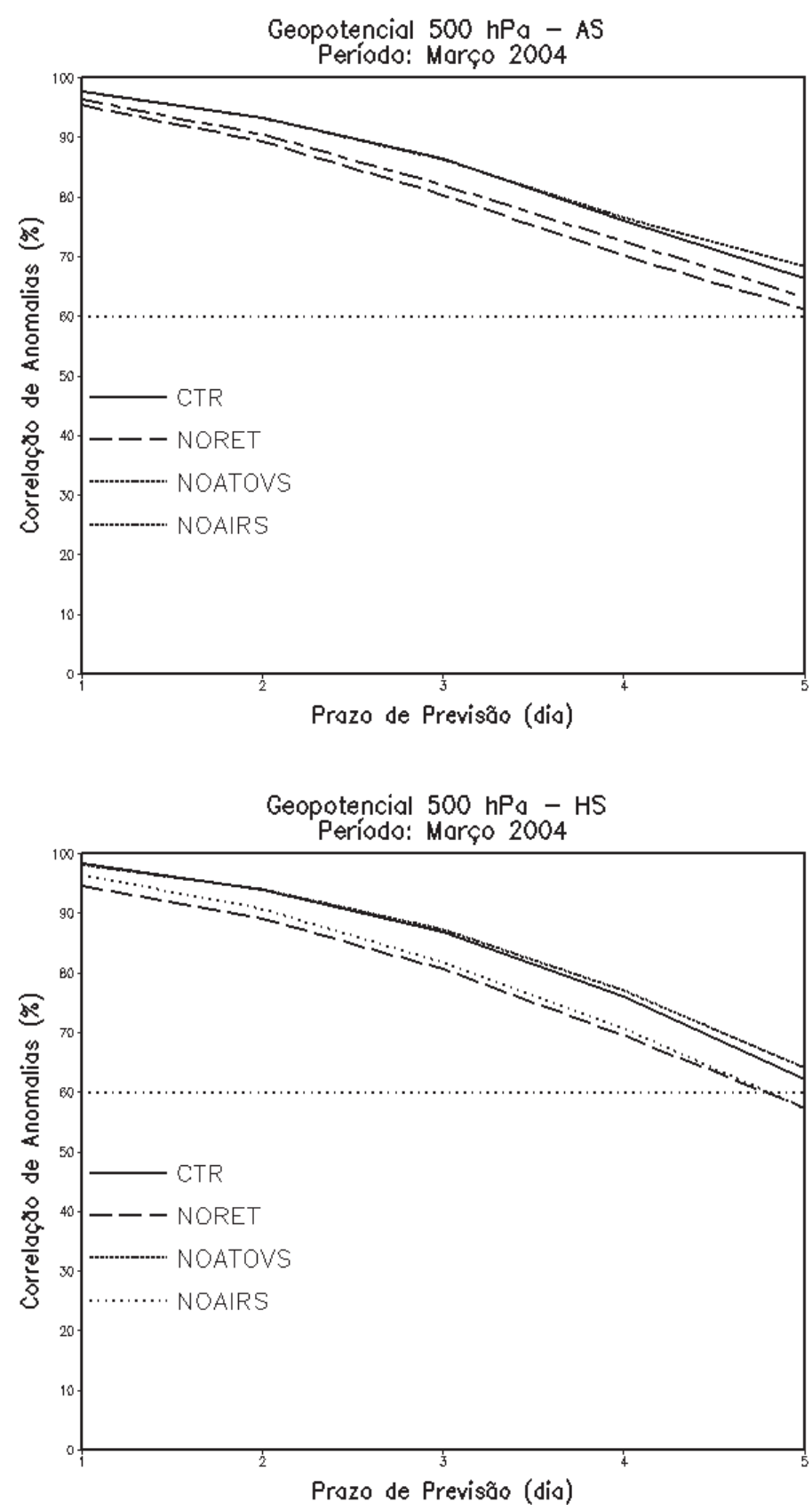

um impacto mais significativo para as previsões de curto prazo, enquanto o impacto do geopotencial, na definição dessa variável, é maior para previsão a partir de 3 dias. Em relação aos campos de umidade específica em 850 hPa para as previsões de 24, 72 e 120 horas (Figura 10), o maior impacto ocorre nas primeiras horas de previsão, com valores de EMQ em torno de 1,5 g/kg para o experimento NOSR4. Os erros referentes ao experimento NOTPW atingem valores da ordem
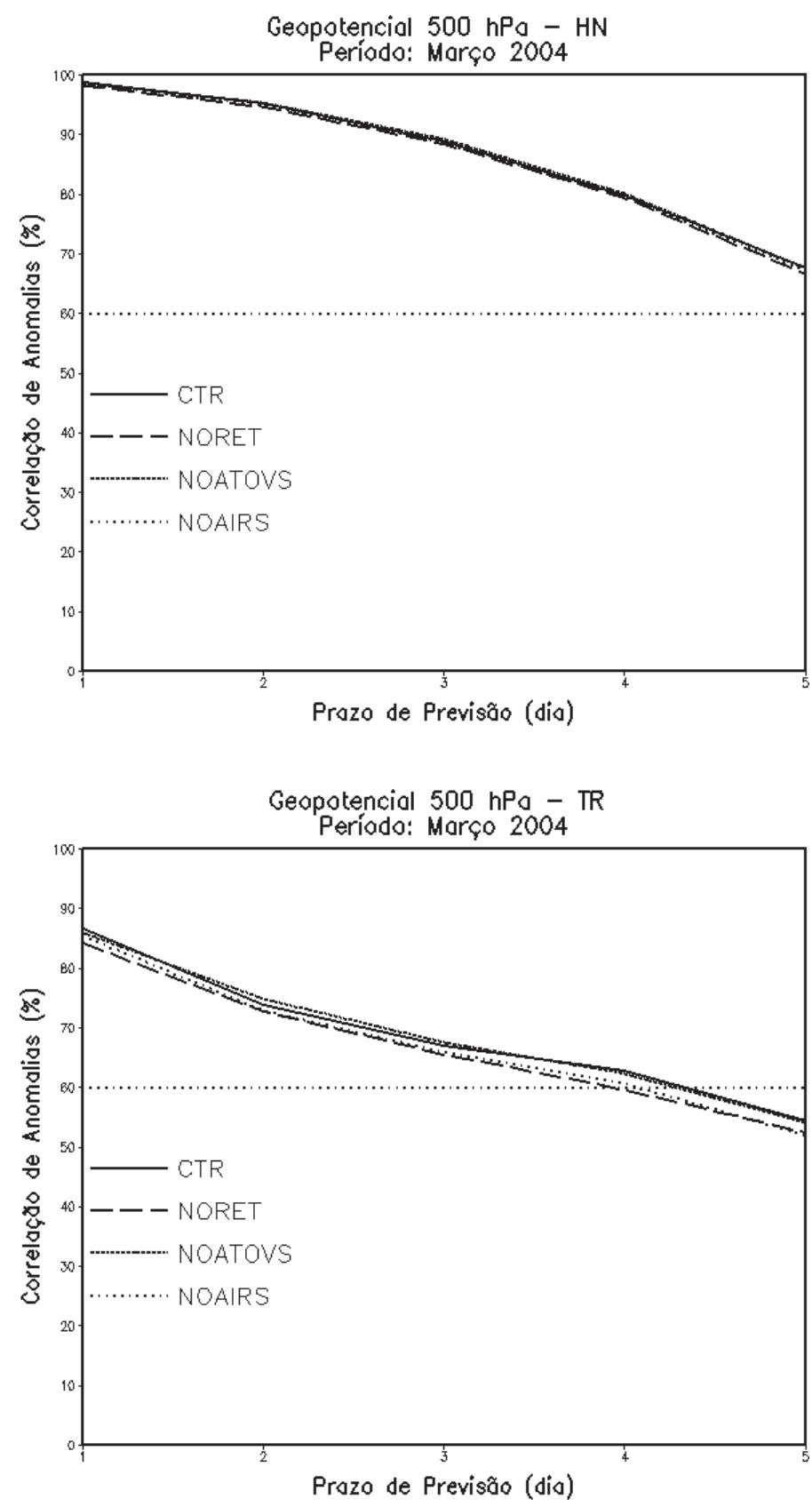

Figura 11 - Idem a Figura 7, exceto para os experimentos CTR, NORET, NOATOVS e NOAIRS. 
de 1,3 g/kg. Observa-se ainda, que para o horário das 24 horas há uma maior variação do EMQ entre os experimentos e que os diversos sistemas de informação afetam o campo de umidade.

Para determinar individualmente o impacto das informações de geopotencial inferidos pelos sistemas de sondagens ATOVS e AIRS/AMSU, na previsão de tempo, foram realizados dois experimentos adicionais, são eles: NOATOVS e NOAIRS. Os resultados desses experimentos são apresentados
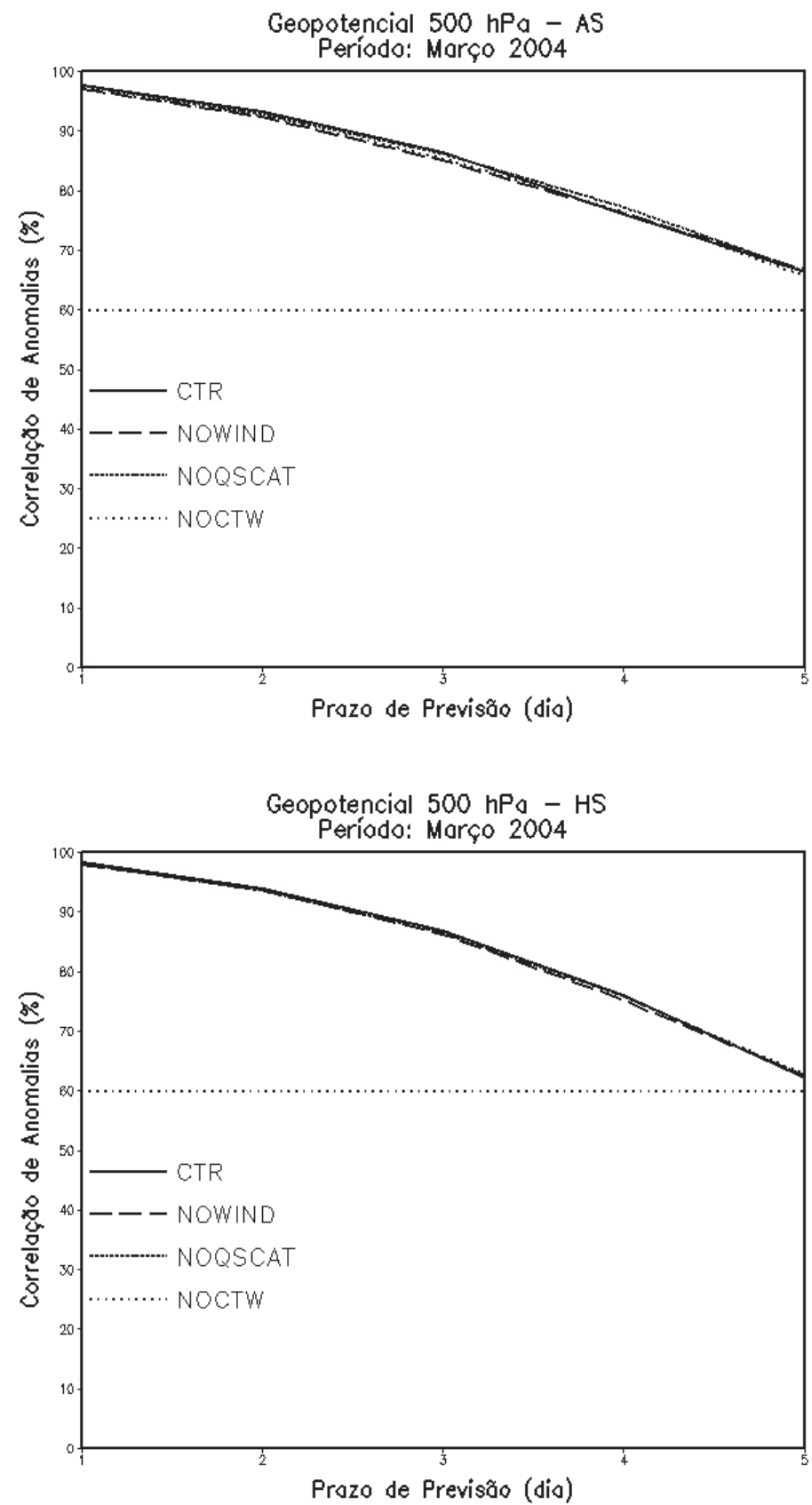

na Figura 11. Exceto para a região do HN, onde o impacto dos dados de satélite é pouco significativo, os resultados para as regiões do HS, tropical e América do Sul mostram que os perfis inferidos pelo sistema de sondagem AIRS/AMSU têm um impacto maior do que àqueles obtidos pelo sistema de sondagem ATOVS. Esta discrepância pode estar associada ao fato de que os perfis atmosféricos AIRS/AMSU são inseridos no ciclo de assimilação como uma observação independente do
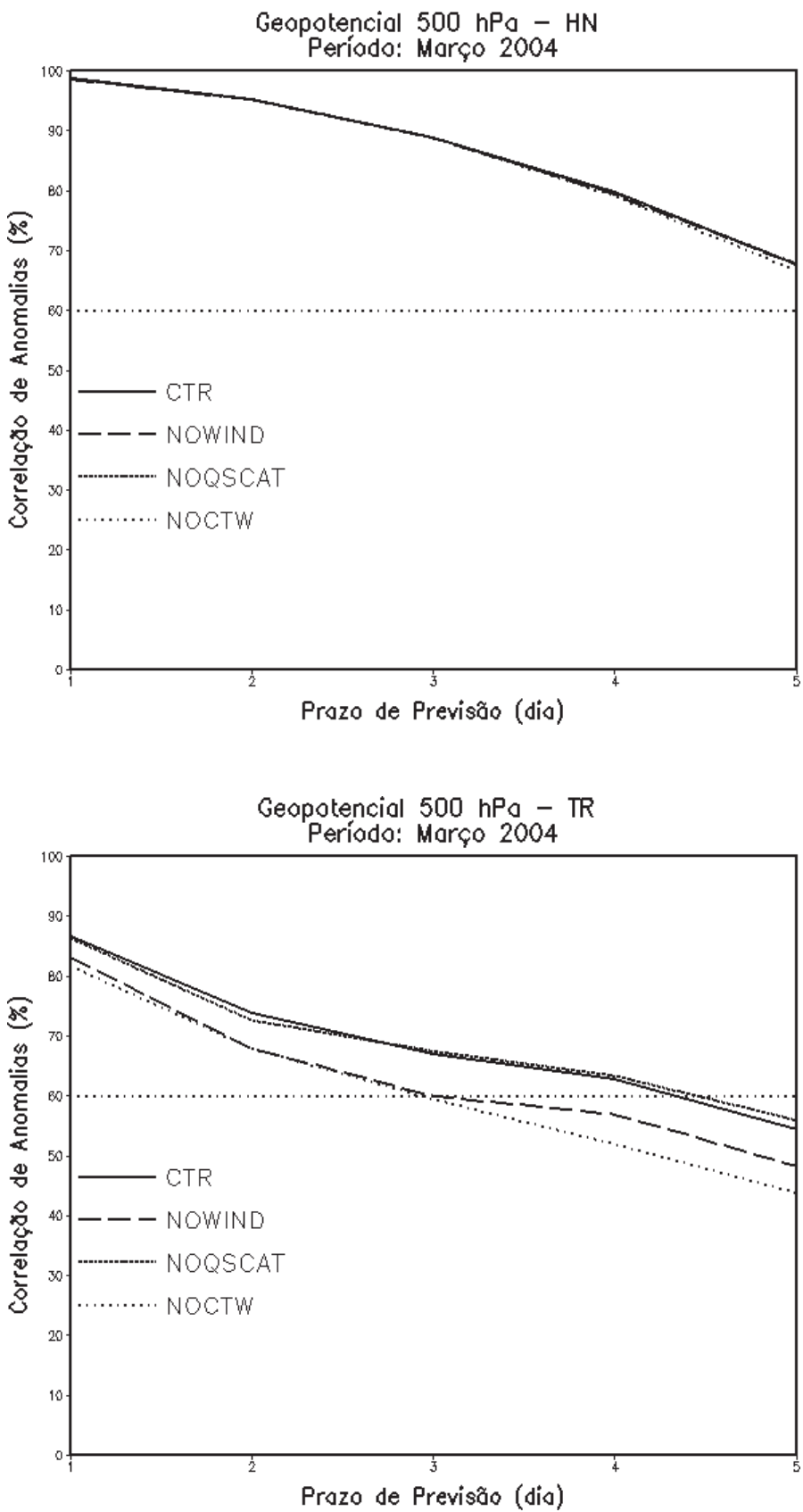

Figura 12 - Idem a Figura 7, exceto para os experimentos CTR, NOWIND, NOQSCAT e NOCTW. 

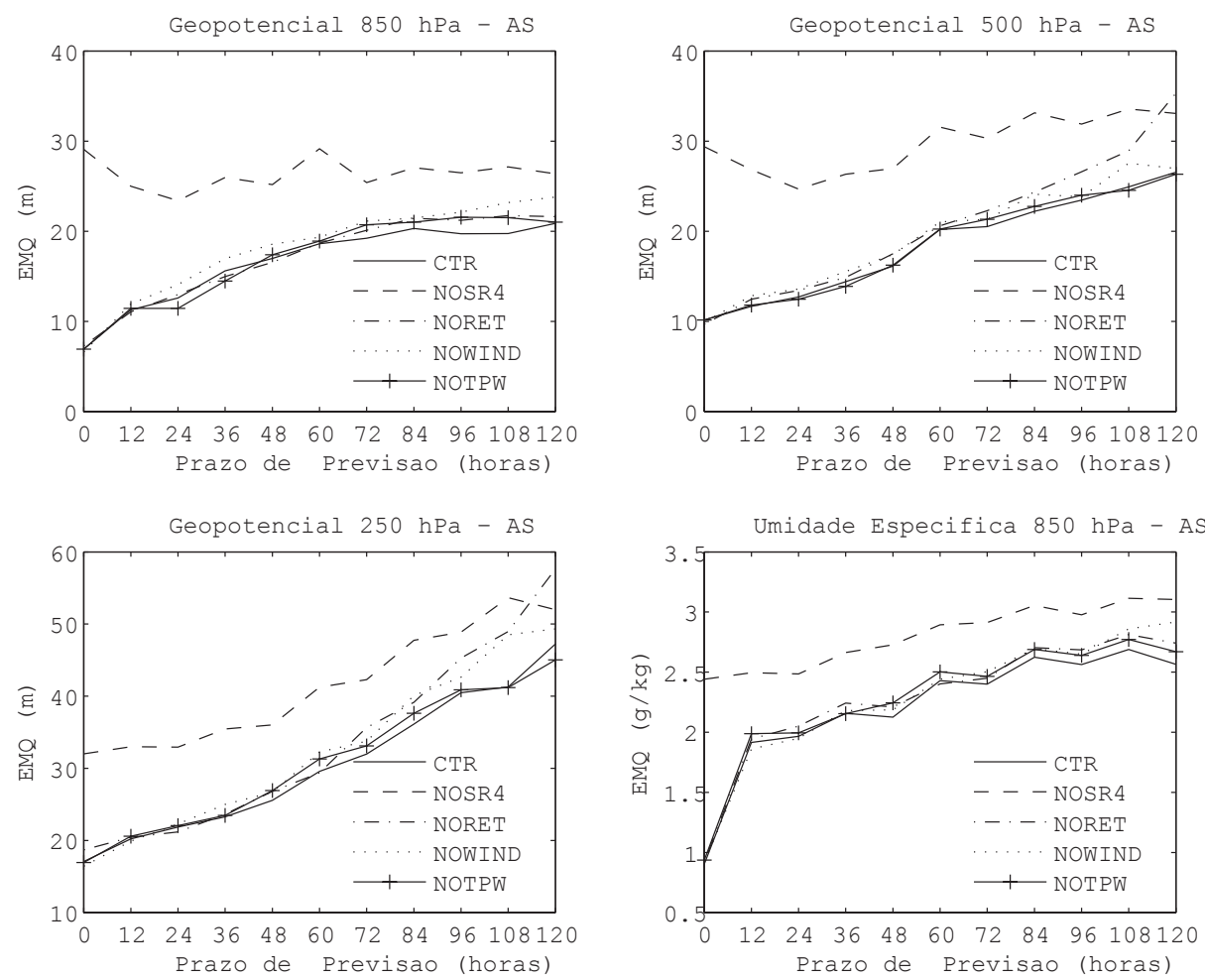

Figura 13 - Média temporal do erro médio quadrático para as previsões do geopotencial em 850,500 e 250 hPa e para a umidade específica em $850 \mathrm{hPa}$, sobre a América do Sul, utilizando como referência dados de radiossondagem no horário das 12 UTC.
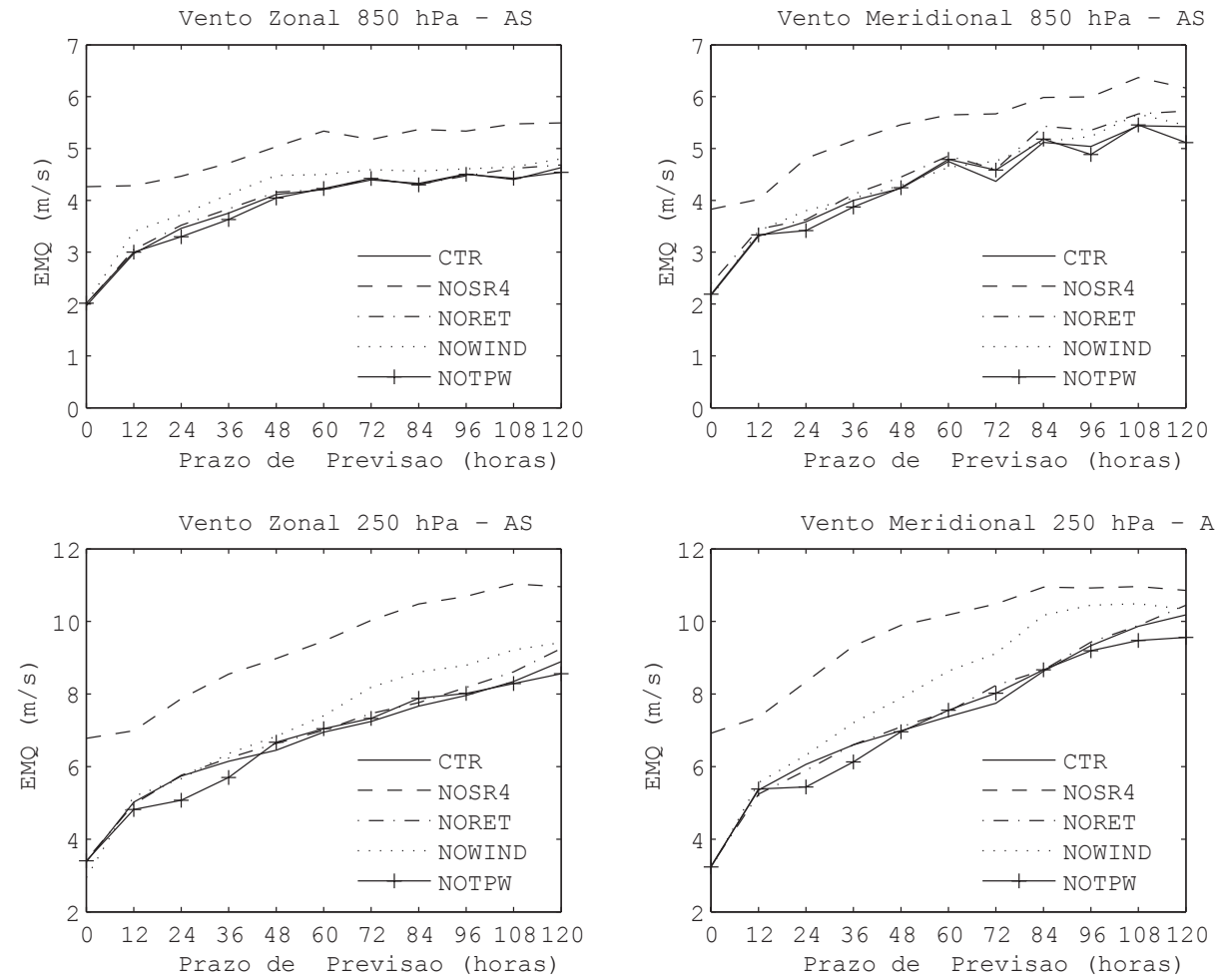

Figura 14 - Idem a Figura 13, exceto para as componentes zonal e meridional do vento em 850 e $250 \mathrm{hPa}$. 
modelo, ou seja, estes perfis não são ancorados no first-guess do modelo como ocorre com os perfis ATOVS. Uma descrição do processo de ancoragem para perfis ATOVS pode ser encontrada em Ferreira (2003). Além disso, a qualidade dos perfis verticais inferidos por satélites tem melhorado significativamente nos últimos anos. Em relação à qualidade dos perfis de temperatura inferidos pelo ATOVS, os perfis AIRS/AMSU mostram uma melhora de no mínimo $0,5 \mathrm{~K}$ (para uma camada vertical de $1 \mathrm{~km}$ ) quando comparados com os dados de radiossondagem (Divakarla et al., 2006). Os experimentos NOQSCAT e NOCTW foram realizados para avaliar o impacto individual das observações de vento estimadas por satélite. A Figura 12 mostra os coeficientes de Correlação de Anomalias do geopotencial em 500 hPa para o MGCA correspondentes a esses experimentos. Nesta figura, nota-se que um impacto significativo ocorre somente sobre a região tropical, como demostrado na Figura 7, e que a remoção dos dados de vento estimados a partir do deslocamento de nuvens (NOCTW) tem um maior impacto sobre a remoção dos dados de vento do QuikScat (NOQSCAT). A importância relativa do CTW pode estar associada às distribuições horizontal e vertical das informações assimiladas. No caso do experimento NOQSCAT, são excluídas no processo de assimilação apenas informações de vento em superfície sobre o oceano. Por outro lado, como mencionado anteriormente, os dados de vento CTW são assimilados nas camadas entre a superfície e 700 $\mathrm{hPa}$ e acima de $400 \mathrm{hPa}$. Além disso, a cobertura espacial dos ventos (CTW) abrange uma extensão quase global na região tropical para todos os horários de análise $(0,6,12$ e 18 UTC) enquanto que as informações de vento do QuikScat restringemse às áreas relativas à passagem do satélite de órbita polar.

Em adição aos cálculos anteriores, que consideram o experimento de CTR como referência, as Figuras 13 e 14 apresentam os valores da raiz do erro médio quadrático para a altura geopotencial em 850,500 e $250 \mathrm{hPa}$, e umidade específica em $850 \mathrm{hPa}$ (Figura 13) e para as componentes zonal e meridional do vento em 850 e $250 \mathrm{hPa}$ (Figura 14), obtidos em relação ao campo observado (radiossondas) sobre a América do Sul, considerando apenas a condição inicial das 12 UTC para os experimentos CTR, NOSR4, NORET, NOWIND e NOTPW. Os maiores erros no geopotencial ocorrem em $850 \mathrm{hPa}$ para o experimento NOSR4, seguido pelo experimento que exclui as informações de vento por satélite (NOWIND). Por outro lado, as informações de massa (experimento NORET) têm um impacto mais significativo na diminuição do erro quando analisamos o geopotencial em 500 e $250 \mathrm{hPa}$. Para esses níveis, nota-se que a exclusão dos perfis atmosféricos ATOVS e AIRS (NORET) gera um maior erro em relação a exclusão dos dados de vento (NOWIND). Para a umidade específica, verifica-se um ligeiro aumento do erro em relação ao experimento de controle, para todos os experimentos. Isto significa que a exclusão de alguma das observações utilizadas no experimento de controle afeta a qualidade dessa variável. Para as componentes zonal e meridional do vento (Figura 14), nota-se, claramente, a importância das informações de vento por satélite para redução dos erros, principalmente em altos níveis, a partir de 36 horas de previsão. É importante ressaltar que esses resultados são baseados em análises pontuais sobre áreas continentais da América do Sul, onde diariamente estão disponíveis dados de radiossondagem. Nesses casos, as informações de satélite sobrepõem tais observações e devido a sua qualidade inferior, as informações de radiossondagem definem, localmente, os campos de análise e previsão. Consequentemente, o maior impacto ocorre para o experimento NOSR4, enquanto que o impacto das outras observações pode ser mascarado.

\section{CONCLUSÕES}

O impacto de diversos sistemas de observações no sistema de assimilação de dados/previsão de tempo do CPTEC é avaliado para o mês de março de 2004. Os experimentos confirmam que existe um impacto benéfico na qualidade dos campos de análises e previsões, quando se utiliza um completo conjunto de observações. Além disso, os resultados mostram que o acerto das previsões geradas pelo sistema GPSAS é fortemente dependente da disponibilidade dos dados, ou seja, em regiões onde existe uma grande quantidade de dados convencionais, como é o caso do HN, o maior impacto nas previsões dessa região ocorre quando esses dados são excluídos do processo de assimilação. Por outro lado, em regiões onde existe uma escassez de observações convencionais tal como o HS (20S-80S), principalmente pelo domínio de áreas oceânicas, os dados de satélite são fundamentais para uma boa previsão. Para a região da América do Sul, dados de satélite contribuem com a mesma ordem de grandeza dos dados convencionais. Isto pode estar associado à melhoria na qualidade dos produtos estimados por satélites em conjunto com a baixa densidade de observações convencionais, além do fato que nos oceanos adjacentes, Pacífico e Atlântico, a análise é determinada principalmente pelas informações de satélites.

Dentre as informações de satélite utilizadas, o maior impacto negativo nas previsões do geopotencial sobre a América do Sul (Figura 7) ocorre quando os perfis atmosféricos AIRS/ AMSU são excluídos do processo de assimilação. Mostrou-se ainda que no caso do vento, o impacto dessa informação estendese para as previsões de curto e médio prazo, no entanto, esse impacto é menor do que aquele obtido quando as informações de vento, estimados por satélite (NOWIND), são excluídas do processo de assimilação para as previsões de curto prazo. Nota-se também que o impacto da exclusão de alguns sistemas de observação, como por exemplo, os ventos em superfície 
sobre o oceano (QuikScat) parecem ser pequenos, porém, esse impacto precisa ser melhor avaliado, principalmente, quando se considera estudos de casos de eventos sinóticos. Em relação ao campo de umidade, observou-se que em regiões com maior disponibilidade de radiossondagens, como no caso do $\mathrm{HN}$, os perfis verticais de umidade oriundos desse sistema de observação dominam na definição dos campos de análises e previsões. Por outro lado, nas regiões tropicais e da América do Sul, as informações de TPW dominam devido à escassez de informações convencionais (umidade específica) nessas regiões.

De forma geral, esses resultados concordam com estudos anteriores realizados em diversos centros de previsão, particularmente, no que diz respeito a importância das observações de satélites meteorológicos. Porém, desde que o número de observações usado varia consideravelmente de um centro para o outro, a importância relativa dos diversos sistemas de observação também pode variar. No entanto, é importante ressaltar que os resultados desses experimentos, em particular, sobre as regiões do HS e América do Sul, não devem ser interpretados de modo a diminuir a relevância dos dados convencionais em relação à quantidade crescente de dados de satélite. A importância das radiossondagens, por exemplo, em previsões obtidas a partir de modelos globais, é confirmada em diversos estudos (Dumelow, 2004; Bouttier e Kelly, 2001). Além disso, apesar das melhorias alcançadas na qualidade dos dados remotos, as observações convencionais são ainda necessárias em regiões onde aquelas são menos precisas, como ocorre em baixos níveis e sob condições de nebulosidade.

Outros experimentos estão sendo realizados com o intuito de avaliar se existe uma dependência temporal (mensal ou sazonal) no desempenho dos experimentos. Além disso, estudos de casos de eventos extremos, baseados nas simulações numéricas realizadas com os diferentes experimentos, serão realizados a fim de avaliar a performance das previsões dos mesmos; principalmente em relação a posição, intensidade e estruturas de ciclones. Finalmente, estudos dessa natureza possibilitam um melhor entendimento de como utilizar as informações disponíveis de forma mais eficiente, as quais são essenciais para o aprimoramento da qualidade das análises e das previsões numéricas de tempo geradas operacionalmente no CPTEC.

\section{AGRADECIMENTOS}

Os autores agradecem o Conselho Nacional de Desenvolvimento Científico e Tecnológico - CNPq (Processo 476129/2006-0) e a Fundação de Amparo à Pesquisa do Estado de São Paulo - FAPESP (Processo 06/53277-6) pelo apoio financeiro à pesquisa.

\section{REFERÊNCIAS BIBLIOGRÁFICAS}

ANDREOLI, R. V.; SOUZA, R. F. A.; FERREIRA, S. F. S.; SAPUCCI, L. F.; HERDIES, D. L.; ARAVÉQUIA, J. A.; MATTOS, J. G. F. First results from the use oh the AQUA sounding system in the CPTEC global data assimilation/ forecast system. In: The 15th International TOVS Study Conference, Maratea, 2006.

ATLAS, R.; HOFFMAN, R. N.; LEIDNER, S. M.; SIENKIEWICZ, J.; YU, T.-W.;. BLOOM, S. C.; BRIN, E.; ARDIZZONE, J.; TERRY, J.; BUNGATO, D.; JUSEM, J. C. The effects of marine winds from scatterometer data on weather analysis and forecasting. Bull. Amer. Meteor. Soc.,v. 82, p.1965- 1990, 2001.

BLOOM, S., DA SILVA, A., DEE, D., et al. Documentation and Validation of the Goddart Earth Observing System (GEOS) Data Assimilation System - Version 4. Technical Report Series on Global Modeling and Data Assimilation. NASA/TM-2005-104606, v. 26, 2005.

BOUTTIER, F.; KELLY, G. Observing-system experiments in the ECMWF 4D-Var data assimilation system. Quart. J. Roy. Meteor. Soc., v. 127, p. 1469-1488, 2001.

CAVALCANTI, I. F. A.; HERDIES, D. L. Data Assimilation Study using SALLJEX Data. CLIVAR Exchanges, Southampton - UK, v. 29, n. 9, p. 23-25, 2004.

CAVALCANTI, I. F. A; MARENGO, J. A.; SATYAMURTY, P.; NOBRE, C.; TROSNIKOV, I.; BONATTI, J. P.; MANZI, A. O., TARASOVA, T.; PEZZI, L. P.; D'ALMEIDA, C.; SAMPAIO, G.; CASTRO, C.; SANCHES, M. B.; CAMARGO, H. Global climatological features in a simulation using the CPTEC-COLA AGCM. J. Climate, v.15, n.21, p. 2965-2988, 2002.

CHEN, S.-H.; VANDENBERGHE, F.; PETTY, G. W.; BRESCH, J. F. Application of SSM/I satellite data to a hurricane simulation. Quart. J. Roy. Meteor. Soc., v.130, p. 801-825, 2004.

CINTRA, R. S. C.; LIMA, W. F.; ESPINOZA, E. S.; CAMPOS VELHO, H. F. Inclusão de dados ATOVS no sistema de assimilaçõa de dados GPSAS do CPTEC. In: XII Simpósio Brasileiro de Sensoriamento Remoto, Goiânia, Brasil, 2005. Anais.

COHN, S. E., DA SILVA, A.; GUO, J.; SIENKIEWICZ, M.; LAMICH, D. Assessing the effects of the data selection with the DAO physical-space statistical analysis system. Mon. Wea. Rev., v.126, p. 2913-2926, 1998.

COURTIER, P.; ANDERSON, E.; HECKLEY, W.; PAILLEUX, J.; VASILJEVIC, D.; HAMRUD, M.; HOLLINGSWORTH, A.; RABIER, F.; FISHER, M. The ECMWF implementation 
of three-dimensional variational assimilation (3D-Var). I:

Formulation. Quart. J. Roy. Meteor. Soc., v. 124, n. 550, p. 1783, 1998.

DIVAKARLA, M. G.; BARNET, C. D.; GOLDBERG, M. D.; MCMILLIN, L. M.; MADDY, E.; WOLF, W.; ZHOU, L.; LIU, X. Validation of atmospheric Infrared Sounder temperature and water vapor retrievals with matched radiosonde measurements and forecasts. J. Geophys. Res., 111, D09S15, doi:10.1029/2005JD006116, 2006.

DUMELOW, R. Overview of observing system experiments. In: ECMWF Seminar Proceedings. Seminar on Recent developments in data assimilation for atmosphere and ocean, European Centre for Medium-Range Weather Forecasts, Shinfield Park, Reading, UK, 8-12 September 2003.

FERREIRA, S. H. S. Análise do procedimento de assimilação de sondagens derivadas de satélites e de seu impacto na previsão de tempo utilizando um sistema estatístico em espaço físico. São José dos Campos, 117p. Dissertação de Mestrado (INPE-11482-TDI/956), 2003.

FERREIRA, S. H. S.; ARAVEQUIA, J. A. A sensibility study of the inclusion of the cloud drift wind data in the CPTEC global data assimilation system. In: 8th International Conference on Southern Hemisphere Meteorology and Oceanography, Foz do Iguaçu, 2006.

HERDIES, D. L.; FERREIRA, S. H.; BONATTI, J. P.; CINTRA, R.; DA SILVA, A. O sistema de assimilação de dados atmosféricos global do CPTEC/INPE. [CD ROM]. In: XII Congresso Brasileiro de Meteorologia, Foz do Iguaçu - PR, 2002. Anais.

KELLY, G., MCNALLY, T.; THEPAUT, J.; SZYNDEL, M. OSE of all main data types in the ECMWF operational system. In: Third WMO Workshop on the Impact of various observing Systems on Numerical Weather Prediction, WMO, Austria, 9-12, March, 2004, Proceedings.

KRISHNAMURTI, T. N.; RAJENDRAN, K.; VIJAYA KUMAR, T. S. V.; LORD, S.; TOTH, Z.; ZOU, X.; COCKE, S.; AHLQUIST, J. E.; NAVON, I. M. Improved Skill for the Anomaly Correlation of Geopotential Heights at $500 \mathrm{hPa}$,
Mon. Weather Rev., v. 131, p. 1082-1102, 2003.

LEDVINA, D. V.; PFAENDTNER, J. Inclusion of SSM/I total precipitable water estimates into the GOES-1 data assimilation system. Mon. Wea. Rev., v. 123, p.3003-3015, 1995.

LEIDNER, S. M.; HOFFMAN, R. N.; AUGENBAUM, J. Sea Winds Scatterometer real-time BUFR Geophysical data product. 45f. User's Guide Version 2.3.0, NOAA/NESDIS, 2000.

MCTAGGART-COWAN R.; BOSART, L. F.; DAVIS, C. A.; ATALLAH, E. H.; GYAKUM, J. R.; EMANUEL, K. A. Analysis of Hurricane Catarina (2004). Mon. Wea. Ver., v. 134, n.11. p. 3029-3053, 2006.

PAILLEUX, J. (Ed.).Impact of Various Observing Systems on Numerical Weather Prediction, Proceedings of CGC/WMO Workshop, Geneva, 7-9 April 1997, WMO Technical Report No. 18, WMO/TD No. 868, 1997.

PARRISH, D. F.; DERBER, J. C. The National Meteorological Center's Spectral Statistical Interpolation Analysis System. Mon. Wea. Rev., v.120, p. 1747-1763, 1992.

SAPUCCI, L. F; ARAVÉQUIA, J. A.; SOUZA, R. A. F.; FERREIRA, S. F. S.; HERDIES, D. L.; ANDREOLI, R. V.; MATTOS, J. G. F. The inclusion of integrated water vapor estimates from AIRS/AMSU and SSM/I sensors into PSAS data assimilation system in the CPTEC. In: The 15th International TOVS Study Conference, Maratea, 2006.

VERNER, G.; SARRAZIN, R.; ZAITSEVA, Y. Data impact studies in the CMC global NWP system. In: Third WMO Workshop on the Impact of various observing Systems on Numerical Weather Prediction, WMO, Austria, 9-12, March, 2004, Proceedings.

WENTZ, F. J.; MATTOX L. A.; PETEHERYCH, S. New algorithms for microwave measurements of ocean winds: Applications to SEASAT and SSM/I. J. Geophys. Res., v. 91, p. 2289-2307, 1986.

ZAPOTOCNY, T. H., MENZEL, W. P.; JUNG, J. A.; NELSON III, J. P. A Four-Season impact study of rawinsonde, GOES, and POES data in the ETA Data Assimilation system. Part II: Contribution of the Components. Wea. Forecasting, v. 20, n. 2, p. 178-198, 2005. 